\title{
Online Advertising Strategies to Effectively Market a Business School
}

\author{
Vikram Kumar ${ }^{1}$, Ramakrishnan Raman ${ }^{1} \&$ R Meenakshi ${ }^{2}$ \\ ${ }^{1}$ Symbiosis International (Deemed University) and Symbiosis Institute of Business Management, Pune, India \\ ${ }^{2}$ Chennai Institute of Technology, Anna University, Chennai, India \\ Correspondence: Ramakrishnan Raman, Symbiosis International (Deemed University) and Symbiosis Institute of \\ Business Management, Pune, India.
}

Received: January 21, 2021

Accepted: February 7, 2021

Online Published: February 9, 2021

doi:10.5430/ijhe.v10n4p61

URL: https://doi.org/10.5430/ijhe.v10n4p61

\begin{abstract}
Advertising has always played an important role in creating visibility for educational institutions. In today's time, digital marketing is the sought-after mode as there has been a significant shift from offline to online advertising. With the evolving times, flexibility and convenience take significant importance and it is critical for educational institutions to shift gears and adapt to the new formats. In order to stay relevant and have a competitive advantage, digital advertising helps higher educational institutions go that extra mile in engaging with their potential customers. It also helps in building awareness and attract good quality of students. In the world of digital advertising, 'Google Advertisement' is an online advertising platform developed by Google, where advertisers bid to display brief advertisements, service offerings, product listings, or videos to web users. It can place advertisements both in the results of search engines like Google Search and on non-search websites, mobile apps, and videos. Google AdWords offers the most pragmatic solutions and tools to all strategic issues of digital advertising. Click Through Ratio (CTR) stands out as the most significant index of reflecting its influence and impact. Amongst the array of choices, the right strategy requires an academic and strategic backing.

The objective of this paper is to assess on the impact of Google Adwords is used in digital advertising campaigns promoting business schools in specific. This research concentrates on CTR as a measure of the campaign's effectiveness. This paper try's to understand CTR in the context related to the type of content embedded in these digital advertisements; the structure of this content; and hence identify and suggest new strategies. This paper identifies and proposes the right online advertising strategy that can be used by a Business School (B School).

Purposive/non-probabilistic sampling was carried out to choose the specific of Business Schools (B-schools) for this study. The business schools selected were based on the National Institution Ranking Framework (NIRF) 2018 of the Indian Human Resource Development. The data was analyzed using to the Social Sciences Statistical Suite (SPSS). There was only access to publicly available and publicly displayed advertisement with no access to user profile data. CTR was utilized to measure total and proportional engagement. The advertisements were then categorized based on their content and analyzed through a one-way ANOVA test. For the purpose of an operationalizing, CTR was utilized as defined by Pak et. al. (2018): "A ratio showing how often people who see your advertisement end up clicking it." The main components analyzed are the characteristics of an effective advertisement appearing on the digital platform measured through its Click Through Ratio. One-way ANOVA has been conducted to assess the Click Through Ratio of advertisement segregated in twenty categories based on their format, content and time of appearance. The analysis reflects that Click Through Ratio differs for different format of advertisements, the information that they contain and for the time and day that they appear. Strategies based on these findings are suggested along with discussion, limitations and further scope of research.
\end{abstract}

Keywords: digital marketing, click through ratio, marketing B schools, Indian higher education

\section{Introduction}

The Indian government has allowed for a wide range of Business Schools (or B Schools) to emerge in all parts of India. Combining private and government institutes, there are over 3000 management institutes in the country. In a country of 1.3 billion people, this is of course not a big number. While certain B Schools come with a strong lineage or decades of experience, others which are relatively new have to prove their presence and attract students to join them. They have to be innovative to showcase their ability to add value to the students. 
Some of the most prestigious destinations for management students include the Indian Institute of Management in Calcutta and the Institute of Management in Ahmedabad. Apart from being established in 1900, they are managed by a government body making them much more credible. They are also associated with some of the global prime institutions like Sloan School of Management (MIT) and Harvard Business School respectively. Private schools have also mushroomed in many Indian cities in the recent past and are establishing their expertise through different and unique methods. Apart from the traditional methods, institutions are also recognizing, accepting and using digital advertising. Marketing and advertising has always played a critical role in building awareness, visibility and brand recall. They have also proved to be effective if conducted in the right way. (Bush et al., 1998) However, Indian higher educational institutions are slowly moving from snail mails, campus visits and brochures, which were the traditional methods to interest candidates to digital advertising . (Hayes et al., 2009) They are now tapping into novel opportunities that can engage with potential customers as well as promote their offerings. A term which now not new to many Indian educational institutions today is 'Digital Advertising'. It was recently found that the major target market of these business schools is a 46 percentage of the total internet users (Keelery, 2020). With tough competition and reducing attention spans, the pressure on all businesses and entities to survive the digital world is very high. (Martín-Santana \& Beerli-Palacio, 2012; Robinson et al.,2007)

Although still in its nascent stages, digital advertising is a dynamic industry which is evolving every single minute. More efficient tools that can be used to promote the business are being introduced regularly. Google Adwords has become one of the strongest contenders which is educating and equipping marketers to experiment, explore and implement their learning. It also offers a vast variety of services on one single account. Google Adwords includes multiple types of digital marketing tools like a banner advertisement, keywords marketing, search engine optimization, search engine marketing, etc.

Utilizing these free and paid resources to their optimum, Business Schools have started harnessing the power of promotion and marketing. There are a wide range of topics that affect the metrics as well multiple methods of quantification of the results. Click Through Ratio (CTR) happens to be one of the more important tools that will be discussed in this research. CTR has analytical capabilities that allows the owner to critically examine and scrutinize what works for the market, the effectiveness of each campaign, timings and days that can be followed and the efficacy of every online advertisement. Apart from dwelling upon the Click Through Rate, this research also aims to find and suggest strategies that can be implemented using the CTR of a campaign.

\section{Literature Review}

\subsection{Postgraduate Education in India}

Education system of India comprises of - primary, secondary or tertiary. It is complicated but dynamic. The recent changes made to the Indian education system (by the National Education Policy 2020) has completely redefines the country's stand on holistic education which bears witness to the evolving nature of Indian education. Likewise, the higher education system functions based on various university classifications granted by the government-run bodies. Universities are mainly classified into Deemed-to-be Universities and Institutes of National Importance, State Universities, Central Universities and Private Universities. This categorization is carried out based on the authority and degree of control of the governing bodies of that institution. Along with this classification, the professional education is largely pronounced by the specific associations or councils such as the Bar Council of India governing the education of law, Medical Council of India (MCI) pronouncing the medicine and doctorate education. There exist fifteen such councils for professional and skill-based education. The overarching body that concerns the decision-making for the majority of the higher education in India is the University Grants Commission (UGC) which is a statutory body established under the Ministry of Human Resource Development by order of the UGC act 1956. The UGC is an integral part of the Indian higher education system as it is responsible for maintaining and strengthening the university education system of the country. Another major characteristic of the Indian higher education system is the accreditation; UGC's National Assessment and Accreditation Council (NAAC) and AICTE's National Board of Accreditation (NBA) are the only two accreditation bodies under the administration of the government. Accreditation from these two councils is the most sought after by universities in India as they hold prestige and increase the credibility of the concerned university. Apart from these, other private accreditation institutions also exist that hold a strong reputation and trust; these include Times Higher Education University Ranking, Quacquarelli Symonds (QS). Specific to business schools, accreditations from the Association to Advance Collegiate Schools of Business (AACSB) and the EFMD Quality Improvement System (EQUIS) governed by the European Foundation for Management Development (EFMD) provide substantially valuable accreditations. With growing numbers of universities and educational institutions opening up every day, having an existence in the 
prospectus of potential students is gaining importance exponentially. Collegiate ranking acts as an integral factor in building this reputation for any higher education institution (Harsha \& Shah, 2011) which is important to attract the right students as they form the backbone of the institution and subsequently the nation. Thank to the National Education Policy 2020, it has been decided that the Higher Education Commission of India (HECI) will act as a single regulator for higher education (barring medical and legal colleges).

\subsection{Indian Business Schools}

The quality of management education provided in Indian universities is improving. The first institution offering a full-time business degree was Andhra University, post which in 1957, the Universities of Kolkata and Delhi introduced their management programs. However, there was no institute entirely dedicated to management education. Indian Institute of Management (IIM) was established in Calcutta in collaboration with the Sloan School of Management of MIT. The next one was set up in Ahmedabad in collaboration with Harvard Business School providing students with all the glory that they bask in today. Later, numerous other institutions were set up in various parts of the country including Lucknow, Indore, Kozhikode (Sinha, 2006) and a large number of private Indian business schools can into existing between 1990 and 1999. The liberalization of the economy was another driver of the high number of B schools in India as the requirement for trained professionals and managers increased due to the establishment of foreign companies. In addition, these business schools also served as a potential investment channel for prospering investors. This further led to a boost of B Schools in the country (Shah, 2012). Based on the statistics of 2019, there are 20 (Indian Institute of Management (IIM) in India and over 3500 B Schools run by various government and private organizations. Due to this, the scenario of business schools in India is rather disheveled. There are numerous private, state, central and private autonomous institutions apart from the institutes that are promoted by large private corporations (Sinha, 2006).

Attracting good students to join B Schools is one of the most difficult tasks. B Schools impart skills and knowledge and also provide the students with an opportunity to join their dream job with a lucrative pay package. A management degree from a prime institution ranks at the top in terms of their yearly salaries barring the administrative and foreign services of India (Philip, 2008). In order to be admitted in any of the 20 IIMs, the candidate has to first appear for the Common Aptitude Test commonly known as CAT which is held yearly. The results are based on percentile patterns, making acceptance and admission more difficult. Likewise, other private institutions with esteemed programs have a test of their own, based on a pattern similar to the CAT; such as SNAP (Symbiosis National Aptitude Test), XAT (Xavier Aptitude Test), NMAT (NMIMS Management Aptitude Test). Almost a hundred million individuals appear for CAT annually along with millions appearing for the various private institute entrance tests.

With the emergence of such a large number of B Schools in the country with the highly competitive admissions process, every institute holds its own merits irrespective of their manner of administration. In this scenario, private B Schools have started to advertise their value offering using online means available. Millions of rupees are spent on the various marketing activities. This is done to show case the brand to the prospective audience. As the B School landscape witnesses the entrance of international institutions as well as international students in Indian business schools, ranking alone does not remain the only influencing factor. Hence, advertising and employing novel promotion \& marketing techniques have also become important. Considering the digital savvy generation who apply to B Schools marketing and promotion online or in the digital arena is utilized by several higher educational institutions (Alexa et al., 2012; Jan \& Ammari, 2016).

\subsection{Digital Advertising and Google AdWords}

As mentioned at the United Nations Summit 2019, the world is growing towards a borderless economy where the digital space will play an important role as it expands platforms for both ends of a business transaction. Hence, advertising is bound to go digital (Mishra, 2020). Martín-Santana \& Beerli-Palacio, (2012) and Robinson et al. (2007) claim that there is not only a need to digitize the advertising process but also feel internal and external pressure to have a digital presence. As a result, digital advertising has become a complex phenomenon with an increasing number of channels and techniques where the compass of success is based on capitalizing on the trends (Girchenko \& Ovsiannikova, 2016). Moreover, as the current generation of millennials are impacted by digital marketing strategies, leading to more frequent visits to the website as well (Smith, 2012). Google AdWords is an advertising tool that is gaining popularity. Google Adwords offers the marketer a comprehensive system to advertise itself on various digital platforms through techniques like keyword planner, Search Engine Optimisation (SEO), Google display network, and advertisement auctions for different kinds of advertisements including display advertisement, banner advertisement, expandable advertisement, video advertisement, and bidding for advertisement 
spaces as well (keyword bidding) (Geddes, 2014). A few models have also been recently developed after assessing the viability of Google Adwords where the different factors influencing the effectiveness of the campaign are quantified (Szymanski \& Lininski, 2018).

Any advertising process proceeds with constant checks, balances and adjustments made based on various results and analysis. The process of digital advertising is no different. As traditional marketing practices are assessed in terms of sales, return on investment, retaining or creating new customers, etc., there are numerous metrics to assess digital advertising campaigns. In digital advertising the effectiveness of a campaign can also be measured in a more quantifiable manner. Key Performance Indicators (KPI) of various matrices help in measuring the success of a digital advertising campaign. Research suggests that KPI form an integral aspect of any digital advertising campaign as they can optimize the campaigns and serve to be critical when managing and honing marketing campaigns (Chaffey \& Patron, 2012; Flores, 2013). Additionally, it has also been found that understanding the nature of the business and product helps in employing the right analytical performance measurement technique. There exist various models in digital advertising ascribing the purpose, functioning and interaction of its own tools (Saura et al., 2017). The KPIs are further subcategorized into two for better and more accurate measurement and analytics. These are 'qualitative' and 'quantitative' measures. Among these, the Click Through Ratio (CTR) is one that indicates the ratio between the users who clicks on a specific link (typically appearing in an advertainment or marketing email) as compared to those who viewed it. It is a very clear indicator for success as it provides the ratio that ultimately indicates the number of users whom the advertisement persuaded to carry out the intended call to action. Hence, the power and capacity of CTR is immense in terms of the various aspects of the advertisement it elaborates on. These aspects include the different shapes of advertisement displayed on a website, length of the content in the ad, animation in the advertisement, etc. (Martín-Santana \& Beerli-Palacio, 2012; Robinson et al., 2007)

Digital advertising has been proving its effectiveness and importance since its inception in 1990 along with establishing its dominance in the field of advertising. It has become essential for businesses to go digital, irrespective of their nature. In the same way educational institutions have also been forced to create their presence in the digital world as most of their potential admissions are mostly online. Latest statistics report that internet penetration in India is of 50\% in the year 2020 and $45 \%$ of these users belong to the age group of 20-29 years (Keelery, 2020). Hence, analyzing the importance and effectiveness of digital advertising by higher education institutions is essential for the sake of their own betterment.

\subsection{Click Through Ratio: Importance \& Characteristics}

Click Through Rate or Click Through Ratio (CTR) is an important statistic when designing, executing and analyzing a digital advertisement campaign. As mentioned earlier, this single statistic possesses the capacity to translate a variety of information about a specific advertisement and as a result, it has many aspects and characteristics that are further affected by many other variables. Through an extensive survey analysis, it has been found that CTR shares a direct relationship with the effectiveness of a square/rectangle banner advertisement appearing on a blog along with the influencers of this effectiveness being an attitude towards the website, involvement with the product being advertised and the duration of the website visit (Martín-Santana \& Beerli-Palacio, 2012). With regards to online games, the characteristics of an effective banner advertisement includes the presence of adequate information regarding the service/product being offered, no immediate promotional incentive in most parts of the advertisement. Moreover, features such as animation and action phrases does not generate a good CTR. These characteristics belong to the banner advertisements. However, all of this information and inferences were gathered from the CTR itself (Robinson et al., 2007). Hence, reinforcing its importance yet again. The colour scheme of the advertisement can also have an impact on the impressions received. The Click Through Rate in their research on the colour schemes of the banner and box advertisement report the findings that advertisement with a colour scheme having red colour produce higher click through rates as compared to the advertisement that have blue colour. Moreover, it was also found that habituation towards certain advertisements can reduce their effectiveness in terms of its CTR. While designing a digital advertisement as a part of a digital marketing campaign, predicting the CTR is also a necessary aspect as it represents the expected outcomes of the proposed strategy. There exist many models to predict the CTR and one of the most inclusive ones is proposed by Yang et al. (2019) based on learning supplementary representation which takes into consideration the compositional aspect of the advertisement, its relational structure and visual appearance. This structure successfully accommodates the new format of advertisement with richer, complicated and interactive advertisements appearing online. In a mathematical derivative study investigating the factors influencing CTR, it was found that the rate of transfer of an advertisement's promotional information with constantly evolving creative affects CTR. However, too many creatives became counterproductive as they may overpower the important 
information intended to be communicated. Therefore, it was concluded that the rate of 0.58 is most effective (Shi, 2017)

Digital Marketing is a wide dimension with innumerable results of a single search, with the world wide web presenting advertisements every time a user enters his/her choice of words. Therefore, the CTR being a measurement matrix, functions with respect to Search Engine Marketing as well. Research suggests that CTR can be an effective predictor of search engine results and that CTR is higher for advertisement containing expert evidences over casual evidences (Haans et al., 2013). These numerous characteristics of CTR have proven to be an important measure of the impact of a digital advertisement campaign therefore having various applications and uses. Similarly, it can be employed to assess and predict marketing campaigns of all natures including for higher education institutions as well.

\subsection{Digital Marketing and CTR in Higher Education Institutions}

Use of digital advertising by educational institutions has been on the rise and its efficacy on various platforms of the digital medium has also been realized (Kumar \& Raman, 2019). With the increasing audience in the digital space, research provides evidence about the increased importance for not only being present on digital mediums but also for having an effective digital advertisement campaign for best admissions. Moreover, it has also been proven that digital strategies are more effective than traditional ones in case of marketing educational institutions (Arora, 2018). A study analyzing the effectiveness of digital advertising for academic institutions, specifically on Google Adwords and the results indicated that advertising educational institutions or programs is very different from the promotion of other businesses. Hence different strategies need to be employed when creating digital marketing campaigns for educational institutions. These results were obtained through the comparison of CTR through statistical package (Turnbull \& Bright, 2008). Kusumawati (2019) reports that digital media is an important element of the student's decision-making process of which course to select. It has been found that students rely heavily on various digital platforms to gather information regarding the various offerings along with providing a direct channel of communication for the candidates that can prove to be helpful in the decision-making process. In a comparative study between a company and an international educational institution, a framework including the various touch points for its digital advertisement campaign has been formulated where CRM was pointed out to be an important element in the campaign (Fierro et al., 2017).

Management institutions or B Schools have a different value and career prospectus attached to them making their viability also different. A few existing pieces of literature have only been initial and exploratory in nature suggesting that there is a considerable improvement required in understanding the customers of business schools and the current digital marketing fails to do so (Nicholls et al., 1995). The research specific to digital marketing of B Schools has been very less and with emerging tools, there is an immediate need for further investigation of these strong novel means. Hence, this study aims at filling this aperture in literature.

\section{Research Objectives}

There are numerous researches conducted on the efficacy of various digital marketing tools of Google Adwords in the stream of how they affect brands and companies. However, very little research has been done on educational institutions and B schools. The objective of this study is to assess how Google Adwords is used in digital marketing advertisement campaigns promoting business schools in specific. Moreover, this research concentrates on CTR as a measure of the campaign's effectiveness. This paper tries to understand CTR in the context related to the type of content embedded in these digital advertisements; the structure of this content; and hence identify and suggest new strategies.

\section{Methodology}

Purposive/non-probabilistic sampling was carried out to choose the specific of Business Schools (B-schools) for this study. The business schools selected were based on the National Institution Ranking Framework (NIRF) 2018 of the Indian Human Resource Development. The data was analyzed using to the Social Sciences Statistical Suite (SPSS). The only access was to the publicly available and the publicly displayed advertisement with no access to user profile data. CTR was utilized to measure total and proportional engagement. The advertisements were then categorized based on their content and analyzed through a one-way ANOVA test. For the purpose of an operationalizing, CTR was be utilized as defined by Pak et. al. (2018): "A ratio showing how often people who see your advertisement end up clicking it."

\section{Analysis}

To understand which category increases click through ratio, the hypothesis was created in the following fashion for 
all the categories. One-way ANOVA (Analysis of Variance) test was used to understand if the click through ratio differed based on the category of the advertisement, as the categories were twenty in number. The categories (twenty) and format (twelve) were created after analyzing all the types of online advertisements posted by B Schools. The value of click through ratio showing how often people who see your advertisement end up clicking it. Click through ratio can be used to measure how well your keywords and advertisement are performing.

Table 1. Results of categorization

\begin{tabular}{|c|c|c|c|}
\hline S,No & Type & $\begin{array}{c}\mathrm{N} \text { (Number of } \\
\text { advertisement) }\end{array}$ & Percentage \\
\hline \multicolumn{4}{|c|}{ Category } \\
\hline 1 & Admissions & 2567 & $7.73 \%$ \\
\hline 2 & Academic & 2045 & $6.16 \%$ \\
\hline 3 & Alumni & 3304 & $9.95 \%$ \\
\hline 4 & Placement related information & 2459 & $7.41 \%$ \\
\hline 5 & Promotion of Programmes & 5943 & $17.90 \%$ \\
\hline 6 & B School Ranking & 2199 & $6.62 \%$ \\
\hline 7 & Clubs and Committees & 316 & $0.95 \%$ \\
\hline 8 & Academic Achievement - Staff & 198 & $0.60 \%$ \\
\hline 9 & Academic Achievement - Faculty member & 278 & $0.84 \%$ \\
\hline 10 & Academic Achievement- Student & 3690 & $11.12 \%$ \\
\hline 11 & B School Competition & 1649 & $4.97 \%$ \\
\hline 12 & Campus Information and updates & 1283 & $3.86 \%$ \\
\hline 12 & Case Competition by B School & 163 & $0.49 \%$ \\
\hline 14 & Case Competition by Corporate & 1657 & $4.99 \%$ \\
\hline 15 & Entertainment and Events & 2672 & $8.05 \%$ \\
\hline 16 & Innovation & 311 & $0.94 \%$ \\
\hline 17 & Internationalization & 329 & $0.99 \%$ \\
\hline 18 & News related to Education & 1066 & $3.21 \%$ \\
\hline 19 & Research Related Information & 379 & $1.14 \%$ \\
\hline \multirow[t]{3}{*}{20} & Startups Information & 690 & $2.08 \%$ \\
\hline & Total & 33198 & $100.00 \%$ \\
\hline & Format & & \\
\hline 1 & Text & 3324 & $10.01 \%$ \\
\hline 2 & Carousel banner & 1815 & $5.47 \%$ \\
\hline 3 & HTML five advertisement & 1963 & $5.91 \%$ \\
\hline 4 & Interactive video & 3153 & $9.50 \%$ \\
\hline 5 & Short video & 4286 & $12.91 \%$ \\
\hline 6 & Long Video & 1907 & $5.75 \%$ \\
\hline 7 & Desktop banner & 3873 & $11.67 \%$ \\
\hline 8 & Mobile banner & 4972 & $14.98 \%$ \\
\hline 9 & Dynamic banner & 2729 & $8.22 \%$ \\
\hline 10 & Text and phone no & 1467 & $4.42 \%$ \\
\hline
\end{tabular}




\begin{tabular}{llcc}
$\mathbf{1 1}$ & static banner & 1823 & $5.49 \%$ \\
$\mathbf{1 2}$ & Extension & 1885 & $5.68 \%$ \\
& Total & 33198 & \\
\hline
\end{tabular}

Table 2. Descriptive information of categories of advertisements

\begin{tabular}{|c|c|c|c|c|c|c|c|c|}
\hline \multirow{2}{*}{ Category type } & \multirow{2}{*}{$\mathbf{N}$} & \multirow{2}{*}{ Mean } & \multirow{2}{*}{$\begin{array}{c}\text { Std. } \\
\text { Deviation }\end{array}$} & \multirow{2}{*}{ Std. Error } & \multicolumn{2}{|c|}{$\begin{array}{l}\text { 95\% Confidence Interval } \\
\text { for Mean }\end{array}$} & \multirow{2}{*}{ Minimum } & \multirow{2}{*}{ Maximum } \\
\hline & & & & & $\begin{array}{l}\text { Lower } \\
\text { Bound }\end{array}$ & $\begin{array}{l}\text { Upper } \\
\text { Bound }\end{array}$ & & \\
\hline Admissions & 100 & .318724 & .1286860 & .0128686 & .293190 & .344258 & .2085 & .9833 \\
\hline Academic & 100 & .185327 & .0578416 & .0057842 & .173850 & .196804 & .0953 & .2907 \\
\hline Alumni & 100 & 192938 & .0360562 & .0036056 & .185784 & .200092 & .1318 & .2523 \\
\hline $\begin{array}{l}\text { Placement } \\
\text { related } \\
\text { information }\end{array}$ & 100 & .388585 & .1187392 & .0118739 & .365025 & .412145 & .3270 & 1.3448 \\
\hline $\begin{array}{l}\text { Promotion of } \\
\text { Programmes }\end{array}$ & 100 & .149483 & .0307868 & .0030787 & .143374 & .155592 & .0949 & .2266 \\
\hline $\begin{array}{l}\text { B School } \\
\text { Ranking }\end{array}$ & 100 & .372197 & .0525231 & .0052523 & .361775 & .382619 & .3209 & .5733 \\
\hline $\begin{array}{l}\text { Clubs and } \\
\text { Committees }\end{array}$ & 100 & .111083 & $\begin{array}{l}.0273 \\
953\end{array}$ & .0027395 & .105647 & .116519 & .0080 & .1364 \\
\hline $\begin{array}{c}\text { Academic } \\
\text { Achievement } \\
\text { - Staff }\end{array}$ & 100 & .050763 & .0166172 & .0016617 & .047466 & .054060 & .0018 & .0758 \\
\hline $\begin{array}{c}\text { Academic } \\
\text { Achievement } \\
\text { - Faculty } \\
\text { member }\end{array}$ & 100 & .059200 & .0199321 & .0019932 & .055245 & .063155 & .0091 & .0908 \\
\hline $\begin{array}{c}\text { Academic } \\
\text { Achievement } \\
\text { - Student }\end{array}$ & 100 & .125470 & .0302948 & .0030295 & .119459 & .131481 & .0028 & .1491 \\
\hline $\begin{array}{l}\text { B School } \\
\text { Competition }\end{array}$ & 100 & .088033 & .0226693 & .0022669 & .083535 & .092531 & .0011 & .0998 \\
\hline $\begin{array}{c}\text { Campus } \\
\text { Information } \\
\text { and updates }\end{array}$ & 100 & 109417 & .0121003 & .0012100 & .107016 & .111818 & .0662 & .1247 \\
\hline $\begin{array}{c}\text { Case } \\
\text { Competition } \\
\text { by B School }\end{array}$ & 100 & .084780 & .0200967 & .0020097 & .080792 & .088768 & .0029 & .0966 \\
\hline $\begin{array}{c}\text { Case } \\
\text { Competition } \\
\text { by Corporate }\end{array}$ & 100 & .047499 & .0129860 & .0012986 & .044922 & .050076 & .0027 & .0664 \\
\hline $\begin{array}{c}\text { Entertainme } \\
\text { nt and } \\
\text { Events }\end{array}$ & 100 & .097960 & .0407794 & .0040779 & .089868 & .106052 & .0671 & .2865 \\
\hline Innovation & 100 & .219316 & .0184693 & .0018469 & .215651 & .222981 & .1419 & .2440 \\
\hline
\end{tabular}




\begin{tabular}{cccccccccc}
\hline $\begin{array}{c}\text { International } \\
\text { ization }\end{array}$ & 100 & .158159 & .0381551 & .0038155 & .150588 & .165730 & .0111 & .1971 \\
$\begin{array}{c}\text { News related } \\
\text { to Education }\end{array}$ & 100 & .046051 & .0178411 & .0017841 & .042511 & .049591 & .0252 & .1246 \\
$\begin{array}{c}\text { Research } \\
\text { Related } \\
\text { Information }\end{array}$ & 100 & .188535 & .0094075 & .0009407 & .186668 & .190402 & .1770 & .2230 \\
$\begin{array}{c}\text { Startups } \\
\text { Information }\end{array}$ & 100 & .089935 & .0205245 & .0020525 & .085862 & .094008 & .0019 & .0997 \\
$\quad$ Total & 2000 & .154173 & .1113482 & .0024898 & .149290 & .159056 & .0011 & 1.3448 \\
\hline
\end{tabular}

The hypothesis (A) Ho: There is no significant difference in the click through ratio for category of advertisement was crafted to check if there was any significant difference in the click through ratio and its relation with the category of the advertisement. The statistical information is given in Table 2 gives descriptive numerical data, including mean, standard deviation and 95\% confidence intervals for the dependent variable- click through score- for each of the category of the advertisement on Google where all the twenty categories have been listed.

Table 2 is the output of the ANOVA analysis which is used to test the Hypothesis (A) to understand if there is a statistically significant difference between the groups of click through ratio and the category of the advertisement. It can be inferred from the significance value 0.000 (i.e., $p=0.000$ ), which is below 0.05 , that there is a statistically significant difference between click through ratio and category of the Advertisement, which clearly indicates that different advertisements under different categories have a different click trough ratio. To know which of those specific categories differed in the Click Through Ratio post hoc test was used. Table 2 gives the output - the multiple comparisons table. From Table 2, it can be inferred that there is a statistically significant difference in click through ratio in the advertisement that were in the admissions category in comparison with all the other categories $(\mathrm{p}=$ 0.000 ). But there is a statistically no significant difference between the advertisement under the Academic category and the advertisement under Alumni $(\mathrm{p}=1.000)$ and Research Related to Information $(\mathrm{p}=1.000)$. For the advertisement related to Admissions and Innovation content categories, it can be inferred that there is a statistically significant difference in click through ratio in the advertisement that were in these categories in comparison with all other categories $(\mathrm{p}=0.000)$. Table 8 gives the details for all the categories for which there is no statistically significant difference in click through ratio of the advertisement when compared to other categories.

Table 3. ANOVA analysis - category type

ANOVA Category type click through ratio

\begin{tabular}{cccccc}
\hline & $\begin{array}{c}\text { Sum of } \\
\text { Squares }\end{array}$ & df & $\begin{array}{c}\text { Mean } \\
\text { Square }\end{array}$ & F & Sig. \\
\hline Between Groups & 20.144 & 19 & 1.060 & 452.367 & .000 \\
$\begin{array}{c}\text { Within Groups } \\
\text { Total }\end{array}$ & 4.641 & 1980 & .002 & & \\
\hline
\end{tabular}

Table 4. Multiple comparisons of categories

\section{Dependent Variable: click through ratio}

Tukey HSD

$\begin{array}{ll}\text { (I) Category } & \text { (J) Category }\end{array}$

\begin{tabular}{lcc} 
& & $(\mathbf{I}-\mathbf{J})$ \\
\hline Admissions & Academic & $.1333970^{*}$ \\
& Alumni & $.1257860^{*}$ \\
\hline
\end{tabular}

Std. Error

Sig. $\quad 95 \%$ Confidence Interval

Lower Upper

Bound Bound

$\begin{array}{llll}.0068464 & .000 & .109101 & .157693 \\ .0068464 & .000 & .101490 & .150082\end{array}$




\begin{tabular}{|c|c|c|c|c|c|c|}
\hline & $\begin{array}{l}\text { Placement } \\
\text { related } \\
\text { information }\end{array}$ & $-.0698610^{*}$ & .0068464 & .000 & -.094157 & -.045565 \\
\hline & $\begin{array}{l}\text { Promotion of } \\
\text { Programmes }\end{array}$ & $.1692410^{*}$ & .0068464 & .000 & .144945 & 193537 \\
\hline & $\begin{array}{l}\text { B School } \\
\text { Ranking }\end{array}$ & $-.0534730^{*}$ & .0068464 & .000 & -.077769 & -.029177 \\
\hline & $\begin{array}{l}\text { Clubs and } \\
\text { Committees }\end{array}$ & $.2076410^{*}$ & .0068464 & .000 & .183345 & .231937 \\
\hline & $\begin{array}{c}\text { Academic } \\
\text { Achievement - } \\
\text { Staff }\end{array}$ & $.2679610^{*}$ & .0068464 & .000 & .243665 & .292257 \\
\hline & $\begin{array}{c}\text { Academic } \\
\text { Achievement - } \\
\text { Faculty member }\end{array}$ & $.2595240^{*}$ & .0068464 & .000 & .235228 & .283820 \\
\hline & $\begin{array}{c}\text { Academic } \\
\text { Achievement- } \\
\text { Student }\end{array}$ & $.1932540^{*}$ & .0068464 & .000 & .168958 & .217550 \\
\hline & $\begin{array}{c}\text { B School } \\
\text { Competition }\end{array}$ & $.2306910^{*}$ & .0068464 & .000 & .206395 & .254987 \\
\hline & $\begin{array}{l}\text { Campus } \\
\text { Information and } \\
\text { updates }\end{array}$ & $.2093070^{*}$ & .0068464 & .000 & .185011 & .233603 \\
\hline & $\begin{array}{c}\text { Case } \\
\text { Competition by } \\
\text { B School }\end{array}$ & $.2339440^{*}$ & .0068464 & .000 & .209648 & .258240 \\
\hline & $\begin{array}{c}\text { Case } \\
\text { Competition by } \\
\text { Corporate }\end{array}$ & $.2712250^{*}$ & .0068464 & .000 & .246929 & .295521 \\
\hline & $\begin{array}{l}\text { Entertainment } \\
\text { and Events }\end{array}$ & $.2207640^{*}$ & .0068464 & .000 & .196468 & .245060 \\
\hline & Innovation & $.0994080^{*}$ & .0068464 & .000 & .075112 & .123704 \\
\hline & $\begin{array}{l}\text { Internationalizati } \\
\text { on }\end{array}$ & $.1605650^{*}$ & .0068464 & .000 & .136269 & .184861 \\
\hline & $\begin{array}{l}\text { News related to } \\
\text { Education }\end{array}$ & $.2726730^{*}$ & .0068464 & .000 & .248377 & .296969 \\
\hline & $\begin{array}{l}\text { Research Related } \\
\text { Information }\end{array}$ & $.1301890^{*}$ & .0068464 & .000 & .105893 & .154485 \\
\hline & $\begin{array}{l}\text { Startups } \\
\text { Information }\end{array}$ & $.2287890^{*}$ & .0068464 & .000 & .204493 & .253085 \\
\hline \multirow[t]{5}{*}{ Academic } & Admissions & $-.1333970^{*}$ & .0068464 & .000 & -.157693 & -.109101 \\
\hline & Alumni & -.0076110 & .0068464 & 1.000 & -.031907 & .016685 \\
\hline & $\begin{array}{l}\text { Placement } \\
\text { related } \\
\text { information }\end{array}$ & $-.2032580^{*}$ & .0068464 & .000 & -.227554 & -.178962 \\
\hline & $\begin{array}{l}\text { Promotion of } \\
\text { Programmes }\end{array}$ & $.0358440^{*}$ & .0068464 & .000 & .011548 & .060140 \\
\hline & $\begin{array}{l}\text { B School } \\
\text { Ranking }\end{array}$ & $-.1868700^{*}$ & .0068464 & .000 & -.211166 & -.162574 \\
\hline
\end{tabular}


Clubs and

Committees

Academic

Achievement Staff

Academic

Achievement -

Faculty member

Academic

Achievement-

Student

B School

Competition

Campus

Information and updates

Case

Competition by

B School

Case

Competition by

Corporate

Entertainment

and Events

Innovation

Internationalizati

on

News related to

Education

Research Related

Information

Startups

Information

Alumni
.0742440

$.1345640^{*}$

$.1261270^{*}$

$.0598570^{*}$

.0068464

.0068464

0068464

.0068464

.0068464

.0068464

.0068464

.0068464

.0068464

.0068464

.0068464

.0068464

.0068464

Placement related information

Promotion of

Programmes

B School

Ranking

Clubs and

Committees

Academic

Achievement Staff
$-.1956470^{*}$

$-.1257860 *$

0076110

$.0434550^{*}$

$-.1792590^{*}$

$.0818550^{*}$

$.1421750^{*}$
$-.0339890$

.0068464

.0068464

.0068464

.0068464

.0068464
.000

.000

.110268

.158860

000

.101831

.150423

000

.035561

.084153
.000

.076251

.000

.113532

.162124

$.000 \quad .063071$

.111663

$-.009693$

.051464

.002872

.114980

.163572

$\begin{array}{lll}1.000 \quad-.027504 & .021088\end{array}$

.000

.071096

.119688

$\begin{array}{lll}.000 & -.150082 & -.101490\end{array}$

$\begin{array}{lll}1.000 & -.016685 & .031907\end{array}$

$\begin{array}{lll}.000 & -.219943 & -.171351\end{array}$

$.000 \quad .019159 \quad .067751$

.000

$-.203555$

$-.154963$

.000

.057559

.106151

.000

.117879

.166471 


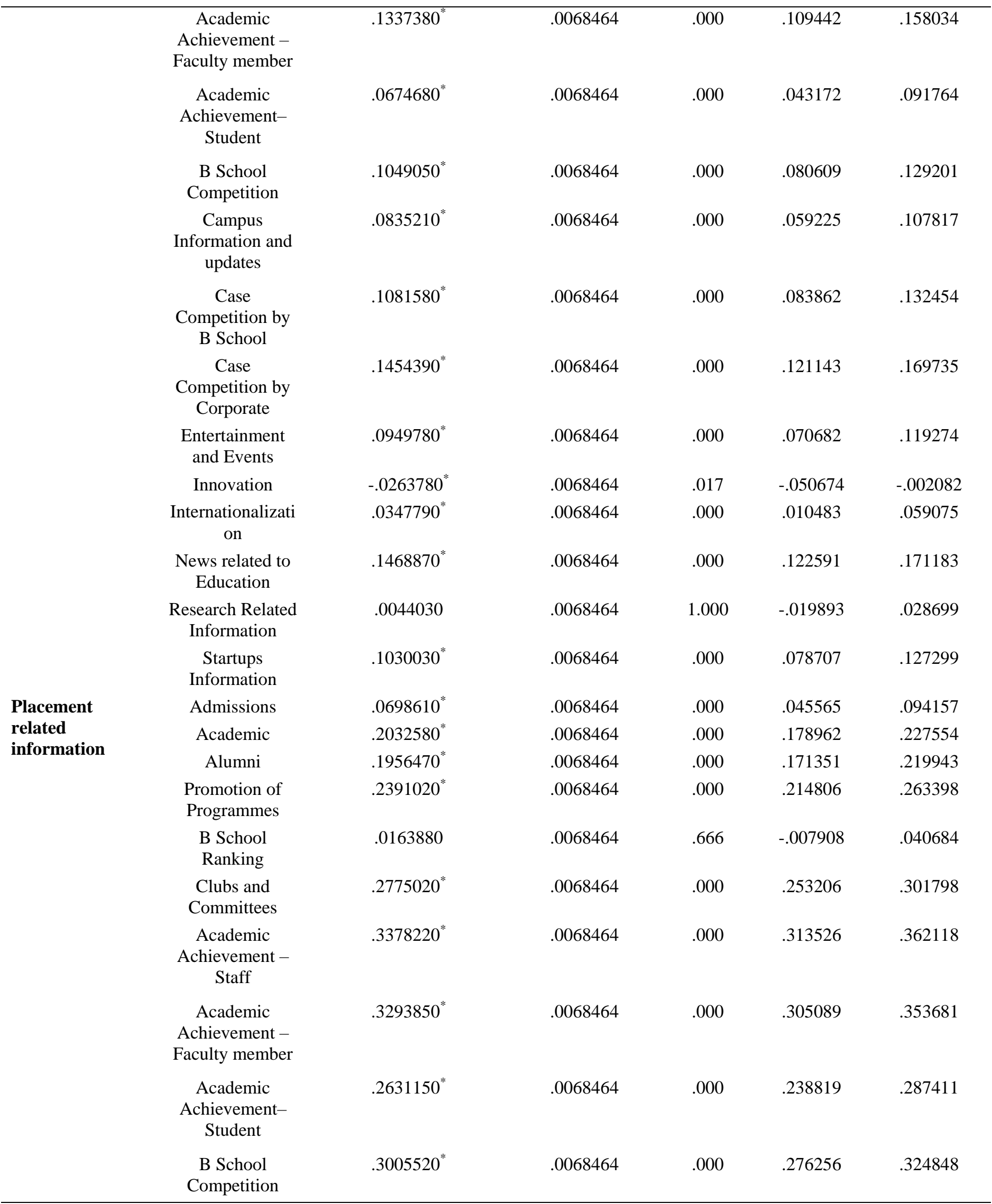




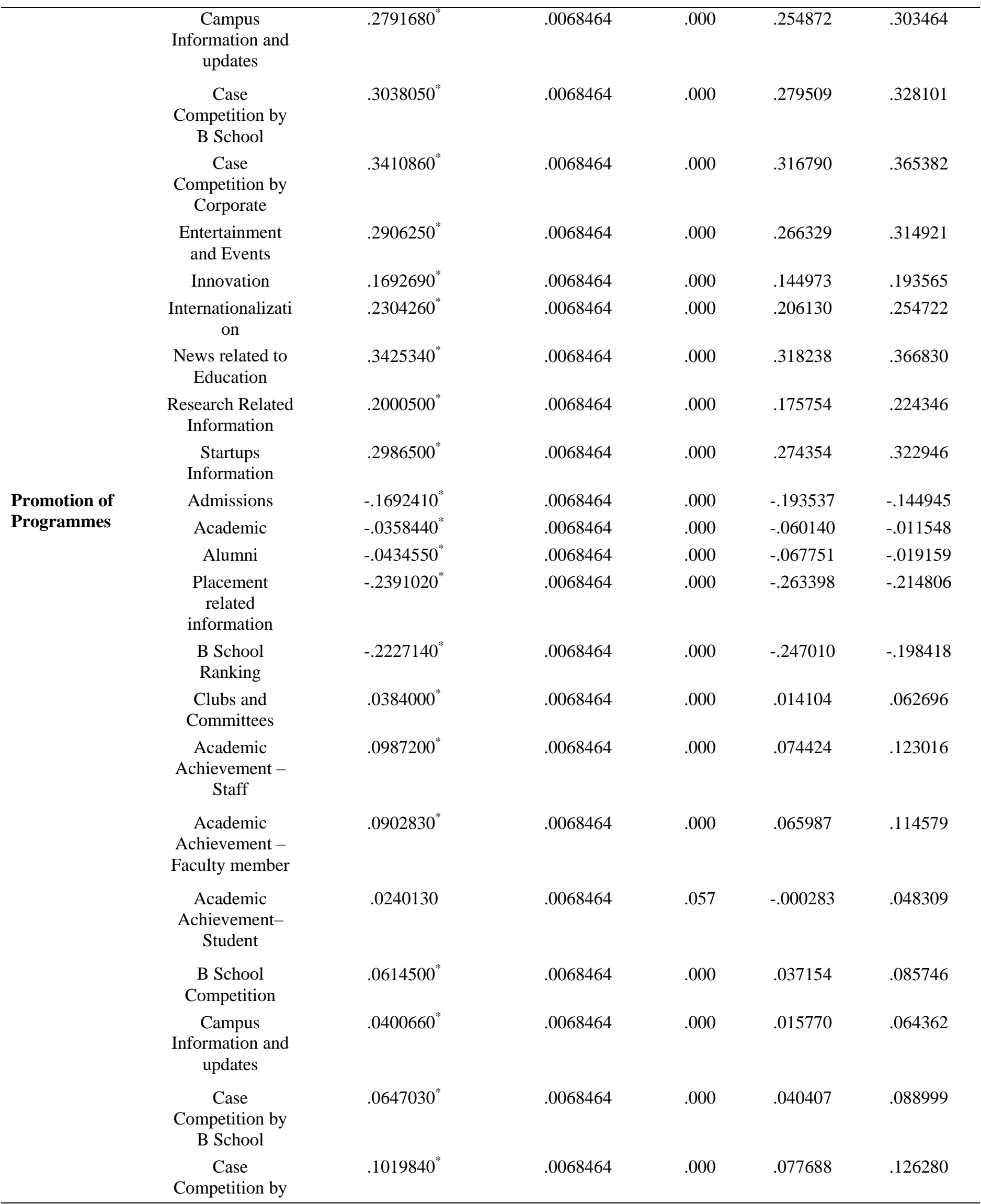


B School

Ranking

\section{Corporate}

Entertainment and Events

Innovation

Internationalizati on

News related to Education

Research Related

Information

Startups Information

Admissions

Academic

Alumni

Placement related

information

Promotion of

Programmes

Clubs and

Committees

Academic

Achievement Staff

Academic

Achievement -

Faculty member

Academic

AchievementStudent

B School

Competition

Campus

Information and updates

Case

Competition by

B School

Case

Competition by Corporate

Entertainment and Events

Innovation

Internationalizati
$.0515230^{*}$

$-.0698330^{*}$

$-.0086760$

$.1034320^{*}$

$-.0390520^{*}$

$.0595480^{*}$

$.0534730^{*}$

$.1868700^{*}$

$.1792590^{*}$

$-.0163880$

$.2227140^{*}$

$.2611140^{*}$

$.3214340^{*}$

$.3129970^{*}$

$.2467270^{*}$

.0068464

$.2841640^{*}$

$.2627800^{*}$

$.2874170^{*}$

$.3246980^{*}$

$.2742370^{*}$

$.1528810^{*}$

$.2140380^{*}$

.0068464

.0068464

.0068464

.0068464

.0068464

.0068464

.0068464

.0068464

.0068464

.0068464

.0068464

.0068464

.0068464

.0068464
.000

.288701

.337293

$\begin{array}{lll}.000 & .029177 & .077769\end{array}$

$.000 \quad .162574$

.154963

$-.040684$

.198418

.247010

.000

.000

.297138

.345730

on 


\begin{tabular}{|c|c|c|c|c|c|c|}
\hline & $\begin{array}{l}\text { News related to } \\
\text { Education }\end{array}$ & $.3261460^{*}$ & .0068464 & .000 & .301850 & .350442 \\
\hline & $\begin{array}{l}\text { Research Related } \\
\text { Information }\end{array}$ & $.1836620^{*}$ & .0068464 & .000 & .159366 & .207958 \\
\hline & $\begin{array}{l}\text { Startups } \\
\text { Information }\end{array}$ & $.2822620^{*}$ & .0068464 & .000 & .257966 & .306558 \\
\hline \multirow{19}{*}{$\begin{array}{l}\text { Clubs and } \\
\text { Committees }\end{array}$} & Admissions & $-.2076410^{*}$ & .0068464 & .000 & -.231937 & -.183345 \\
\hline & Academic & $-.0742440^{*}$ & .0068464 & .000 & -.098540 & -.049948 \\
\hline & Alumni & $-.0818550^{*}$ & .0068464 & .000 & -.106151 & -.057559 \\
\hline & $\begin{array}{l}\text { Placement } \\
\text { related } \\
\text { information }\end{array}$ & $-.2775020^{*}$ & .0068464 & .000 & -.301798 & -.253206 \\
\hline & $\begin{array}{l}\text { Promotion of } \\
\text { Programmes }\end{array}$ & $-.0384000^{*}$ & .0068464 & .000 & -.062696 & -.014104 \\
\hline & $\begin{array}{l}\text { B School } \\
\text { Ranking }\end{array}$ & $-.2611140^{*}$ & .0068464 & .000 & -.285410 & -.236818 \\
\hline & $\begin{array}{c}\text { Academic } \\
\text { Achievement - } \\
\text { Staff }\end{array}$ & $.0603200^{*}$ & .0068464 & .000 & .036024 & .084616 \\
\hline & $\begin{array}{c}\text { Academic } \\
\text { Achievement - } \\
\text { Faculty member }\end{array}$ & $.0518830^{*}$ & .0068464 & .000 & .027587 & .076179 \\
\hline & $\begin{array}{c}\text { Academic } \\
\text { Achievement- } \\
\text { Student }\end{array}$ & -.0143870 & .0068464 & .856 & -.038683 & .009909 \\
\hline & $\begin{array}{l}\text { B School } \\
\text { Competition }\end{array}$ & .0230500 & .0068464 & .088 & -.001246 & .047346 \\
\hline & $\begin{array}{c}\text { Campus } \\
\text { Information and } \\
\text { updates }\end{array}$ & .0016660 & .0068464 & 1.000 & -.022630 & .025962 \\
\hline & $\begin{array}{c}\text { Case } \\
\text { Competition by } \\
\text { B School }\end{array}$ & $.0263030^{*}$ & .0068464 & .018 & .002007 & .050599 \\
\hline & $\begin{array}{c}\text { Case } \\
\text { Competition by } \\
\text { Corporate }\end{array}$ & $.0635840^{*}$ & .0068464 & .000 & .039288 & .087880 \\
\hline & $\begin{array}{l}\text { Entertainment } \\
\text { and Events }\end{array}$ & .0131230 & .0068464 & .933 & -.011173 & .037419 \\
\hline & Innovation & $-.1082330^{*}$ & .0068464 & .000 & -.132529 & -.083937 \\
\hline & $\begin{array}{l}\text { Internationalizati } \\
\text { on }\end{array}$ & $-.0470760^{*}$ & .0068464 & .000 & -.071372 & -.022780 \\
\hline & $\begin{array}{l}\text { News related to } \\
\text { Education }\end{array}$ & $.0650320^{*}$ & .0068464 & .000 & .040736 & .089328 \\
\hline & $\begin{array}{l}\text { Research Related } \\
\text { Information }\end{array}$ & $-.0774520^{*}$ & .0068464 & .000 & -.101748 & -.053156 \\
\hline & $\begin{array}{l}\text { Startups } \\
\text { Information }\end{array}$ & .0211480 & .0068464 & .188 & -.003148 & .045444 \\
\hline \multirow{2}{*}{$\begin{array}{l}\text { Academic } \\
\text { Achievement - }\end{array}$} & Admissions & $-.2679610^{*}$ & .0068464 & .000 & -.292257 & -.243665 \\
\hline & Academic & $-.1345640^{*}$ & .0068464 & .000 & -.158860 & -.110268 \\
\hline
\end{tabular}




\begin{tabular}{|c|c|c|c|c|c|c|}
\hline \multirow[t]{17}{*}{ Staff } & Alumni & $-.1421750^{*}$ & .0068464 & .000 & -.166471 & -.117879 \\
\hline & $\begin{array}{l}\text { Placement } \\
\text { related } \\
\text { information }\end{array}$ & $-.3378220^{*}$ & .0068464 & .000 & -.362118 & -.313526 \\
\hline & $\begin{array}{l}\text { Promotion of } \\
\text { Programmes }\end{array}$ & $-.0987200^{*}$ & .0068464 & .000 & -.123016 & -.074424 \\
\hline & $\begin{array}{l}\text { B School } \\
\text { Ranking }\end{array}$ & $-.3214340^{*}$ & .0068464 & .000 & -.345730 & -.297138 \\
\hline & $\begin{array}{l}\text { Clubs and } \\
\text { Committees }\end{array}$ & $-.0603200^{*}$ & .0068464 & .000 & -.084616 & -.036024 \\
\hline & $\begin{array}{c}\text { Academic } \\
\text { Achievement - } \\
\text { Faculty member }\end{array}$ & -.0084370 & .0068464 & 1.000 & -.032733 & .015859 \\
\hline & $\begin{array}{c}\text { Academic } \\
\text { Achievement- } \\
\text { Student }\end{array}$ & $-.0747070^{*}$ & .0068464 & .000 & -.099003 & -.050411 \\
\hline & $\begin{array}{c}\text { B School } \\
\text { Competition }\end{array}$ & $-.0372700^{*}$ & .0068464 & .000 & -.061566 & -.012974 \\
\hline & $\begin{array}{l}\text { Campus } \\
\text { Information and } \\
\text { updates }\end{array}$ & $-.0586540^{*}$ & .0068464 & .000 & -.082950 & -.034358 \\
\hline & $\begin{array}{c}\text { Case } \\
\text { Competition by } \\
\text { B School }\end{array}$ & $-.0340170^{*}$ & .0068464 & .000 & -.058313 & -.009721 \\
\hline & $\begin{array}{c}\text { Case } \\
\text { Competition by } \\
\text { Corporate }\end{array}$ & .0032640 & .0068464 & 1.000 & -.021032 & .027560 \\
\hline & $\begin{array}{l}\text { Entertainment } \\
\text { and Events }\end{array}$ & $-.0471970^{*}$ & .0068464 & .000 & -.071493 & -.022901 \\
\hline & Innovation & $-.1685530^{*}$ & .0068464 & .000 & -.192849 & -.144257 \\
\hline & $\begin{array}{l}\text { Internationalizati } \\
\text { on }\end{array}$ & $-.1073960^{*}$ & .0068464 & .000 & -.131692 & -.083100 \\
\hline & $\begin{array}{l}\text { News related to } \\
\text { Education }\end{array}$ & .0047120 & .0068464 & 1.000 & -.019584 & .029008 \\
\hline & $\begin{array}{l}\text { Research Related } \\
\text { Information }\end{array}$ & $-.1377720^{*}$ & .0068464 & .000 & -.162068 & -.113476 \\
\hline & $\begin{array}{l}\text { Startups } \\
\text { Information }\end{array}$ & $-.0391720^{*}$ & .0068464 & .000 & -.063468 & -.014876 \\
\hline \multirow{6}{*}{$\begin{array}{l}\text { Academic } \\
\text { Achievement - } \\
\text { Faculty member }\end{array}$} & Admissions & $-.2595240^{*}$ & .0068464 & .000 & -.283820 & -.235228 \\
\hline & Academic & $-.1261270^{*}$ & .0068464 & .000 & -.150423 & -.101831 \\
\hline & Alumni & $-.1337380^{*}$ & .0068464 & .000 & -.158034 & -.109442 \\
\hline & $\begin{array}{l}\text { Placement } \\
\text { related } \\
\text { information }\end{array}$ & $-.3293850^{*}$ & .0068464 & .000 & -.353681 & -.305089 \\
\hline & $\begin{array}{l}\text { Promotion of } \\
\text { Programmes }\end{array}$ & $-.0902830^{*}$ & .0068464 & .000 & -.114579 & -.065987 \\
\hline & $\begin{array}{l}\text { B School } \\
\text { Ranking }\end{array}$ & $-.3129970^{*}$ & .0068464 & .000 & -.337293 & -.288701 \\
\hline
\end{tabular}




\begin{tabular}{|c|c|c|c|c|c|c|}
\hline & $\begin{array}{c}\text { Clubs and } \\
\text { Committees }\end{array}$ & $-.0518830^{*}$ & .0068464 & .000 & -.076179 & -.027587 \\
\hline & $\begin{array}{c}\text { Academic } \\
\text { Achievement - } \\
\text { Staff }\end{array}$ & .0084370 & .0068464 & 1.000 & -.015859 & .032733 \\
\hline & $\begin{array}{c}\text { Academic } \\
\text { Achievement- } \\
\text { Student }\end{array}$ & $-.0662700^{*}$ & .0068464 & .000 & -.090566 & -.041974 \\
\hline & $\begin{array}{c}\text { B School } \\
\text { Competition }\end{array}$ & $-.0288330^{*}$ & .0068464 & .004 & -.053129 & -.004537 \\
\hline & $\begin{array}{c}\text { Campus } \\
\text { Information and } \\
\text { updates }\end{array}$ & $-.0502170^{*}$ & .0068464 & .000 & -.074513 & -.025921 \\
\hline & $\begin{array}{c}\text { Case } \\
\text { Competition by } \\
\text { B School }\end{array}$ & $-.0255800^{*}$ & .0068464 & .026 & -.049876 & -.001284 \\
\hline & $\begin{array}{c}\text { Case } \\
\text { Competition by } \\
\text { Corporate }\end{array}$ & .0117010 & .0068464 & .978 & -.012595 & .035997 \\
\hline & $\begin{array}{l}\text { Entertainment } \\
\text { and Events }\end{array}$ & $-.0387600^{*}$ & .0068464 & .000 & -.063056 & -.014464 \\
\hline & Innovation & $-.1601160^{*}$ & .0068464 & .000 & -.184412 & -.135820 \\
\hline & $\begin{array}{l}\text { Internationalizati } \\
\text { on }\end{array}$ & $-.0989590^{*}$ & .0068464 & .000 & -.123255 & -.074663 \\
\hline & $\begin{array}{l}\text { News related to } \\
\text { Education }\end{array}$ & .0131490 & .0068464 & .931 & -.011147 & .037445 \\
\hline & $\begin{array}{l}\text { Research Related } \\
\text { Information }\end{array}$ & $-.1293350^{*}$ & .0068464 & .000 & -.153631 & -.105039 \\
\hline & $\begin{array}{c}\text { Startups } \\
\text { Information }\end{array}$ & $-.0307350^{*}$ & .0068464 & .001 & -.055031 & -.006439 \\
\hline Academic & Admissions & $-.1932540^{*}$ & .0068464 & .000 & -.217550 & -.168958 \\
\hline Achievement- & Academic & $-.0598570^{*}$ & .0068464 & .000 & -.084153 & -.035561 \\
\hline & Alumni & $-.0674680^{*}$ & .0068464 & .000 & -.091764 & -.043172 \\
\hline & $\begin{array}{l}\text { Placement } \\
\text { related } \\
\text { information }\end{array}$ & $-.2631150^{*}$ & .0068464 & .000 & -.287411 & -.238819 \\
\hline & $\begin{array}{l}\text { Promotion of } \\
\text { Programmes }\end{array}$ & -.0240130 & .0068464 & .057 & -.048309 & .000283 \\
\hline & $\begin{array}{l}\text { B School } \\
\text { Ranking }\end{array}$ & $-.2467270^{*}$ & .0068464 & .000 & -.271023 & -.222431 \\
\hline & $\begin{array}{l}\text { Clubs and } \\
\text { Committees }\end{array}$ & .0143870 & .0068464 & .856 & -.009909 & .038683 \\
\hline & $\begin{array}{c}\text { Academic } \\
\text { Achievement - } \\
\text { Staff }\end{array}$ & $.0747070^{*}$ & .0068464 & .000 & .050411 & .099003 \\
\hline & $\begin{array}{c}\text { Academic } \\
\text { Achievement - } \\
\text { Faculty member }\end{array}$ & $.0662700^{*}$ & .0068464 & .000 & .041974 & .090566 \\
\hline
\end{tabular}




\begin{tabular}{|c|c|c|c|c|c|c|}
\hline & $\begin{array}{c}\text { B School } \\
\text { Competition }\end{array}$ & $.0374370^{*}$ & .0068464 & .000 & .013141 & .061733 \\
\hline & $\begin{array}{c}\text { Campus } \\
\text { Information and } \\
\text { updates }\end{array}$ & .0160530 & .0068464 & .703 & -.008243 & .040349 \\
\hline & $\begin{array}{c}\text { Case } \\
\text { Competition by } \\
\text { B School }\end{array}$ & $.0406900^{*}$ & .0068464 & .000 & .016394 & .064986 \\
\hline & $\begin{array}{c}\text { Case } \\
\text { Competition by } \\
\text { Corporate }\end{array}$ & $.0779710^{*}$ & .0068464 & .000 & .053675 & .102267 \\
\hline & $\begin{array}{l}\text { Entertainment } \\
\text { and Events }\end{array}$ & $.0275100^{*}$ & .0068464 & .009 & .003214 & .051806 \\
\hline & Innovation & $-.0938460^{*}$ & .0068464 & .000 & -.118142 & -.069550 \\
\hline & $\begin{array}{l}\text { Internationalizati } \\
\text { on }\end{array}$ & $-.0326890^{*}$ & .0068464 & .000 & -.056985 & -.008393 \\
\hline & $\begin{array}{l}\text { News related to } \\
\text { Education }\end{array}$ & $.0794190^{*}$ & .0068464 & .000 & .055123 & .103715 \\
\hline & $\begin{array}{l}\text { Research Related } \\
\text { Information }\end{array}$ & $-.0630650^{*}$ & .0068464 & .000 & -.087361 & -.038769 \\
\hline & $\begin{array}{l}\text { Startups } \\
\text { Information }\end{array}$ & $.0355350^{*}$ & .0068464 & .000 & .011239 & .059831 \\
\hline \multirow{12}{*}{$\begin{array}{l}\text { B School } \\
\text { Competition }\end{array}$} & Admissions & $-.2306910^{*}$ & .0068464 & .000 & -.254987 & -.206395 \\
\hline & Academic & $-.0972940^{*}$ & .0068464 & .000 & -.121590 & -.072998 \\
\hline & Alumni & $-.1049050^{*}$ & .0068464 & .000 & -.129201 & -.080609 \\
\hline & $\begin{array}{l}\text { Placement } \\
\text { related } \\
\text { information }\end{array}$ & $-.3005520^{*}$ & .0068464 & .000 & -.324848 & -.276256 \\
\hline & $\begin{array}{l}\text { Promotion of } \\
\text { Programmes }\end{array}$ & $-.0614500^{*}$ & .0068464 & .000 & -.085746 & -.037154 \\
\hline & $\begin{array}{l}\text { B School } \\
\text { Ranking }\end{array}$ & $-.2841640^{*}$ & .0068464 & .000 & -.308460 & -.259868 \\
\hline & $\begin{array}{l}\text { Clubs and } \\
\text { Committees }\end{array}$ & -.0230500 & .0068464 & .088 & -.047346 & .001246 \\
\hline & $\begin{array}{c}\text { Academic } \\
\text { Achievement - } \\
\text { Staff }\end{array}$ & $.0372700^{*}$ & .0068464 & .000 & .012974 & .061566 \\
\hline & $\begin{array}{c}\text { Academic } \\
\text { Achievement - } \\
\text { Faculty member }\end{array}$ & $.0288330^{*}$ & .0068464 & .004 & .004537 & .053129 \\
\hline & $\begin{array}{c}\text { Academic } \\
\text { Achievement- } \\
\text { Student }\end{array}$ & $-.0374370^{*}$ & .0068464 & .000 & -.061733 & -.013141 \\
\hline & $\begin{array}{c}\text { Campus } \\
\text { Information and } \\
\text { updates }\end{array}$ & -.0213840 & .0068464 & .172 & -.045680 & .002912 \\
\hline & $\begin{array}{c}\text { Case } \\
\text { Competition by } \\
\text { B School }\end{array}$ & .0032530 & .0068464 & 1.000 & -.021043 & .027549 \\
\hline
\end{tabular}




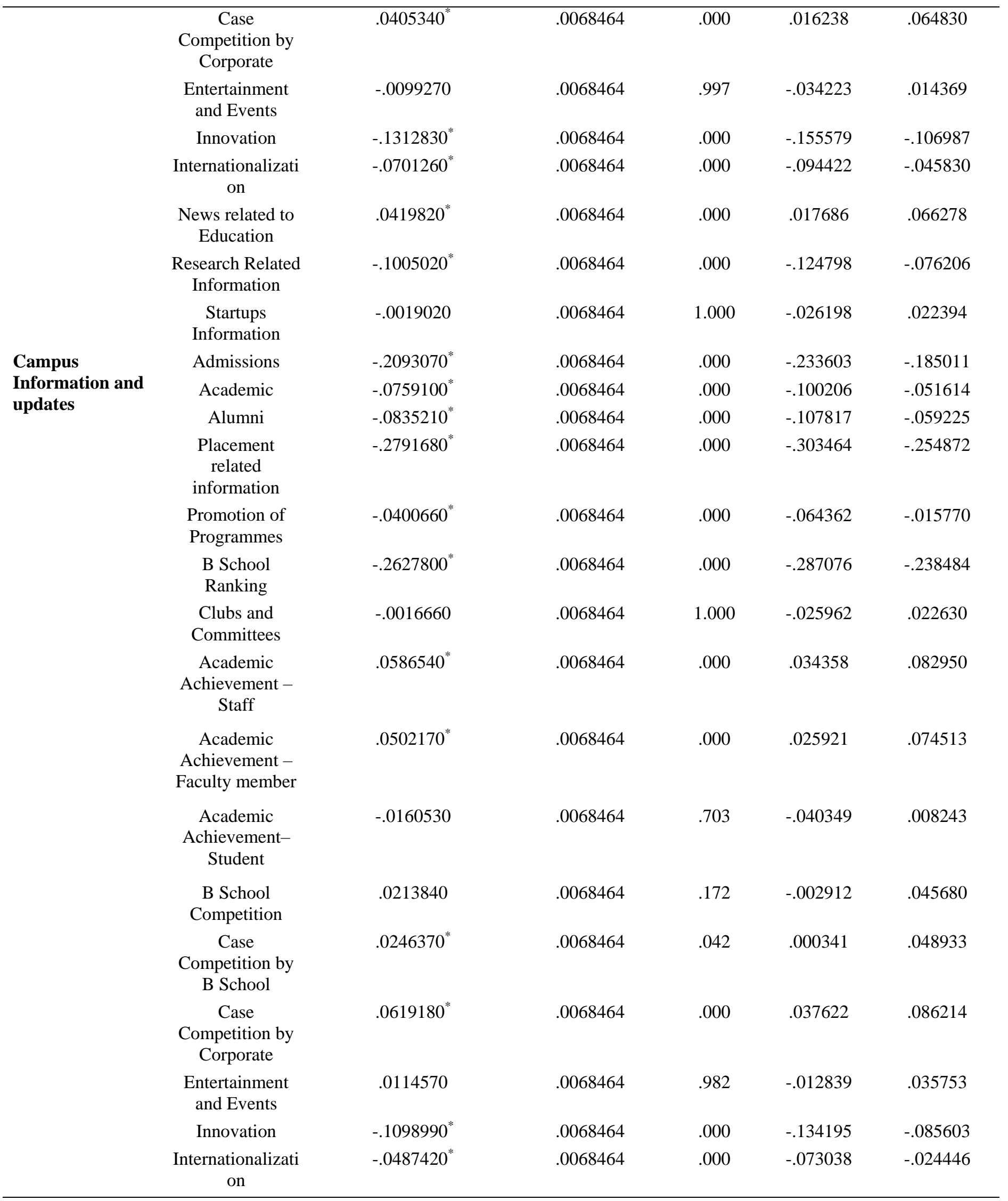




\begin{tabular}{|c|c|c|c|c|c|c|}
\hline & $\begin{array}{l}\text { News related to } \\
\text { Education }\end{array}$ & $.0633660^{*}$ & .0068464 & .000 & .039070 & .087662 \\
\hline & $\begin{array}{l}\text { Research Related } \\
\text { Information }\end{array}$ & $-.0791180^{*}$ & .0068464 & .000 & -.103414 & -.054822 \\
\hline & $\begin{array}{l}\text { Startups } \\
\text { Information }\end{array}$ & .0194820 & .0068464 & .326 & -.004814 & .043778 \\
\hline \multirow{19}{*}{$\begin{array}{l}\text { Case } \\
\text { Competition by } \\
\text { B School }\end{array}$} & Admissions & $-.2339440^{*}$ & .0068464 & .000 & -.258240 & -.209648 \\
\hline & Academic & $-.1005470^{*}$ & .0068464 & .000 & -.124843 & -.076251 \\
\hline & Alumni & $-.1081580^{*}$ & .0068464 & .000 & -.132454 & -.083862 \\
\hline & $\begin{array}{l}\text { Placement } \\
\text { related } \\
\text { information }\end{array}$ & $-.3038050^{*}$ & .0068464 & .000 & -.328101 & -.279509 \\
\hline & $\begin{array}{l}\text { Promotion of } \\
\text { Programmes }\end{array}$ & $-.0647030^{*}$ & .0068464 & .000 & -.088999 & -.040407 \\
\hline & $\begin{array}{l}\text { B School } \\
\text { Ranking }\end{array}$ & $-.2874170^{*}$ & .0068464 & .000 & -.311713 & -.263121 \\
\hline & $\begin{array}{l}\text { Clubs and } \\
\text { Committees }\end{array}$ & $-.0263030^{*}$ & .0068464 & .018 & -.050599 & -.002007 \\
\hline & $\begin{array}{c}\text { Academic } \\
\text { Achievement - } \\
\text { Staff }\end{array}$ & $.0340170^{*}$ & .0068464 & .000 & .009721 & .058313 \\
\hline & $\begin{array}{c}\text { Academic } \\
\text { Achievement - } \\
\text { Faculty member }\end{array}$ & $.0255800^{*}$ & .0068464 & .026 & .001284 & .049876 \\
\hline & $\begin{array}{c}\text { Academic } \\
\text { Achievement- } \\
\text { Student }\end{array}$ & $-.0406900^{*}$ & .0068464 & .000 & -.064986 & -.016394 \\
\hline & $\begin{array}{l}\text { B School } \\
\text { Competition }\end{array}$ & -.0032530 & .0068464 & 1.000 & -.027549 & .021043 \\
\hline & $\begin{array}{l}\text { Campus } \\
\text { Information and } \\
\text { updates }\end{array}$ & $-.0246370^{*}$ & .0068464 & .042 & -.048933 & -.000341 \\
\hline & $\begin{array}{c}\text { Case } \\
\text { Competition by } \\
\text { Corporate }\end{array}$ & $.0372810^{*}$ & .0068464 & .000 & .012985 & .061577 \\
\hline & $\begin{array}{l}\text { Entertainment } \\
\text { and Events }\end{array}$ & -.0131800 & .0068464 & .930 & -.037476 & .011116 \\
\hline & Innovation & $-.1345360^{*}$ & .0068464 & .000 & -.158832 & -.110240 \\
\hline & $\begin{array}{l}\text { Internationalizati } \\
\text { on }\end{array}$ & $-.0733790^{*}$ & .0068464 & .000 & -.097675 & -.049083 \\
\hline & $\begin{array}{l}\text { News related to } \\
\text { Education }\end{array}$ & $.0387290^{*}$ & .0068464 & .000 & .014433 & .063025 \\
\hline & $\begin{array}{l}\text { Research Related } \\
\text { Information }\end{array}$ & $-.1037550^{*}$ & .0068464 & .000 & -.128051 & -.079459 \\
\hline & $\begin{array}{c}\text { Startups } \\
\text { Information }\end{array}$ & -.0051550 & .0068464 & 1.000 & -.029451 & .019141 \\
\hline \multirow{2}{*}{$\begin{array}{l}\text { Case } \\
\text { Competition by }\end{array}$} & Admissions & $-.2712250^{*}$ & .0068464 & .000 & -.295521 & -.246929 \\
\hline & Academic & $-.1378280^{*}$ & .0068464 & .000 & -.162124 & -.113532 \\
\hline
\end{tabular}




\begin{tabular}{|c|c|c|c|c|c|c|}
\hline \multirow[t]{12}{*}{ Corporate } & Alumni & $-.1454390^{*}$ & .0068464 & .000 & -.169735 & -.121143 \\
\hline & $\begin{array}{l}\text { Placement } \\
\text { related } \\
\text { information }\end{array}$ & $-.3410860^{*}$ & .0068464 & .000 & -.365382 & -.316790 \\
\hline & $\begin{array}{l}\text { Promotion of } \\
\text { Programmes }\end{array}$ & $-.1019840^{*}$ & .0068464 & .000 & -.126280 & -.077688 \\
\hline & $\begin{array}{l}\text { B School } \\
\text { Ranking }\end{array}$ & $-.3246980^{*}$ & .0068464 & .000 & -.348994 & -.300402 \\
\hline & $\begin{array}{l}\text { Clubs and } \\
\text { Committees }\end{array}$ & $-.0635840^{*}$ & .0068464 & .000 & -.087880 & -.039288 \\
\hline & $\begin{array}{c}\text { Academic } \\
\text { Achievement - } \\
\text { Staff }\end{array}$ & -.0032640 & .0068464 & 1.000 & -.027560 & .021032 \\
\hline & $\begin{array}{c}\text { Academic } \\
\text { Achievement - } \\
\text { Faculty member }\end{array}$ & -.0117010 & .0068464 & .978 & -.035997 & .012595 \\
\hline & $\begin{array}{c}\text { Academic } \\
\text { Achievement- } \\
\text { Student }\end{array}$ & $-.0779710^{*}$ & .0068464 & .000 & -.102267 & -.053675 \\
\hline & $\begin{array}{c}\text { B School } \\
\text { Competition }\end{array}$ & $-.0405340^{*}$ & .0068464 & .000 & -.064830 & -.016238 \\
\hline & $\begin{array}{c}\text { Campus } \\
\text { Information and } \\
\text { updates }\end{array}$ & $-.0619180^{*}$ & .0068464 & .000 & -.086214 & -.037622 \\
\hline & $\begin{array}{c}\text { Case } \\
\text { Competition by } \\
\text { B School }\end{array}$ & $-.0372810^{*}$ & .0068464 & .000 & -.061577 & -.012985 \\
\hline & $\begin{array}{l}\text { Entertainment } \\
\text { and Events }\end{array}$ & $-.0504610^{*}$ & .0068464 & .000 & -.074757 & -.026165 \\
\hline \multirow{11}{*}{$\begin{array}{l}\text { Entertainment } \\
\text { and Events }\end{array}$} & Innovation & $-.1718170^{*}$ & .0068464 & .000 & -.196113 & -.147521 \\
\hline & $\begin{array}{l}\text { Internationalizati } \\
\text { on }\end{array}$ & $-.1106600^{*}$ & .0068464 & .000 & -.134956 & -.086364 \\
\hline & $\begin{array}{l}\text { News related to } \\
\text { Education }\end{array}$ & .0014480 & .0068464 & 1.000 & -.022848 & .025744 \\
\hline & $\begin{array}{l}\text { Research Related } \\
\text { Information }\end{array}$ & $-.1410360^{*}$ & .0068464 & .000 & -.165332 & -.116740 \\
\hline & $\begin{array}{l}\text { Startups } \\
\text { Information }\end{array}$ & $-.0424360^{*}$ & .0068464 & .000 & -.066732 & -.018140 \\
\hline & Admissions & $-.2207640^{*}$ & .0068464 & .000 & -.245060 & -.196468 \\
\hline & Academic & $-.0873670^{*}$ & .0068464 & .000 & -.111663 & -.063071 \\
\hline & Alumni & $-.0949780^{*}$ & .0068464 & .000 & -.119274 & -.070682 \\
\hline & $\begin{array}{l}\text { Placement } \\
\text { related } \\
\text { information }\end{array}$ & $-.2906250^{*}$ & .0068464 & .000 & -.314921 & -.266329 \\
\hline & $\begin{array}{l}\text { Promotion of } \\
\text { Programmes }\end{array}$ & $-.0515230^{*}$ & .0068464 & .000 & -.075819 & -.027227 \\
\hline & $\begin{array}{l}\text { B School } \\
\text { Ranking }\end{array}$ & $-.2742370^{*}$ & .0068464 & .000 & -.298533 & -.249941 \\
\hline
\end{tabular}




\begin{tabular}{|c|c|c|c|c|c|c|}
\hline & $\begin{array}{c}\text { Clubs and } \\
\text { Committees }\end{array}$ & -.0131230 & .0068464 & .933 & -.037419 & .011173 \\
\hline & $\begin{array}{c}\text { Academic } \\
\text { Achievement - } \\
\text { Staff }\end{array}$ & $.0471970^{*}$ & .0068464 & .000 & .022901 & .071493 \\
\hline & $\begin{array}{c}\text { Academic } \\
\text { Achievement - } \\
\text { Faculty member }\end{array}$ & $.0387600^{*}$ & .0068464 & .000 & .014464 & .063056 \\
\hline & $\begin{array}{c}\text { Academic } \\
\text { Achievement- } \\
\text { Student }\end{array}$ & $-.0275100^{*}$ & .0068464 & .009 & -.051806 & -.003214 \\
\hline & $\begin{array}{c}\text { B School } \\
\text { Competition }\end{array}$ & .0099270 & .0068464 & .997 & -.014369 & .034223 \\
\hline & $\begin{array}{c}\text { Campus } \\
\text { Information and } \\
\text { updates }\end{array}$ & -.0114570 & .0068464 & .982 & -.035753 & .012839 \\
\hline & $\begin{array}{c}\text { Case } \\
\text { Competition by } \\
\text { B School }\end{array}$ & .0131800 & .0068464 & .930 & -.011116 & .037476 \\
\hline & $\begin{array}{l}\text { Case } \\
\text { Competition by } \\
\text { Corporate }\end{array}$ & $.0504610^{*}$ & .0068464 & .000 & .026165 & .074757 \\
\hline & Innovation & $-.1213560^{*}$ & .0068464 & .000 & -.145652 & -.097060 \\
\hline & $\begin{array}{l}\text { Internationalizati } \\
\text { on }\end{array}$ & $-.0601990^{*}$ & .0068464 & .000 & -.084495 & -.035903 \\
\hline & $\begin{array}{l}\text { News related to } \\
\text { Education }\end{array}$ & $.0519090^{*}$ & .0068464 & .000 & .027613 & .076205 \\
\hline & $\begin{array}{l}\text { Research Related } \\
\text { Information }\end{array}$ & $-.0905750^{*}$ & .0068464 & .000 & -.114871 & -.066279 \\
\hline & $\begin{array}{l}\text { Startups } \\
\text { Information }\end{array}$ & .0080250 & .0068464 & 1.000 & -.016271 & .032321 \\
\hline Innovation & Admissions & $-.0994080^{*}$ & .0068464 & .000 & -.123704 & -.075112 \\
\hline & Academic & $.0339890^{*}$ & .0068464 & .000 & .009693 & .058285 \\
\hline & Alumni & $.0263780^{*}$ & .0068464 & .017 & .002082 & .050674 \\
\hline & $\begin{array}{l}\text { Placement } \\
\text { related } \\
\text { information }\end{array}$ & $-.1692690^{*}$ & .0068464 & .000 & -.193565 & -.144973 \\
\hline & $\begin{array}{l}\text { Promotion of } \\
\text { Programmes }\end{array}$ & $.0698330^{*}$ & .0068464 & .000 & .045537 & .094129 \\
\hline & $\begin{array}{l}\text { B School } \\
\text { Ranking }\end{array}$ & $-.1528810^{*}$ & .0068464 & .000 & -.177177 & -.128585 \\
\hline & $\begin{array}{l}\text { Clubs and } \\
\text { Committees }\end{array}$ & $.1082330^{*}$ & .0068464 & .000 & .083937 & .132529 \\
\hline & $\begin{array}{c}\text { Academic } \\
\text { Achievement - } \\
\text { Staff }\end{array}$ & $.1685530^{*}$ & .0068464 & .000 & .144257 & .192849 \\
\hline & $\begin{array}{c}\text { Academic } \\
\text { Achievement - } \\
\text { Faculty member }\end{array}$ & $.1601160^{*}$ & .0068464 & .000 & .135820 & .184412 \\
\hline
\end{tabular}




\begin{tabular}{|c|c|c|c|c|c|c|}
\hline & $\begin{array}{c}\text { Academic } \\
\text { Achievement- } \\
\text { Student }\end{array}$ & $.0938460^{*}$ & .0068464 & .000 & .069550 & .118142 \\
\hline & $\begin{array}{c}\text { B School } \\
\text { Competition }\end{array}$ & $.1312830^{*}$ & .0068464 & .000 & .106987 & .155579 \\
\hline & $\begin{array}{c}\text { Campus } \\
\text { Information and } \\
\text { updates }\end{array}$ & $.1098990^{*}$ & .0068464 & .000 & .085603 & .134195 \\
\hline & $\begin{array}{c}\text { Case } \\
\text { Competition by } \\
\text { B School }\end{array}$ & $.1345360^{*}$ & .0068464 & .000 & .110240 & .158832 \\
\hline & $\begin{array}{c}\text { Case } \\
\text { Competition by } \\
\text { Corporate }\end{array}$ & $.1718170^{*}$ & .0068464 & .000 & .147521 & .196113 \\
\hline & $\begin{array}{l}\text { Entertainment } \\
\text { and Events }\end{array}$ & $.1213560^{*}$ & .0068464 & .000 & .097060 & .145652 \\
\hline & $\begin{array}{l}\text { Internationalizati } \\
\text { on }\end{array}$ & $.0611570^{*}$ & .0068464 & .000 & .036861 & .085453 \\
\hline & $\begin{array}{l}\text { News related to } \\
\text { Education }\end{array}$ & $.1732650^{*}$ & .0068464 & .000 & .148969 & .197561 \\
\hline & $\begin{array}{l}\text { Research Related } \\
\text { Information }\end{array}$ & $.0307810^{*}$ & .0068464 & .001 & .006485 & .055077 \\
\hline & $\begin{array}{l}\text { Startups } \\
\text { Information }\end{array}$ & $.1293810^{*}$ & .0068464 & .000 & .105085 & .153677 \\
\hline \multirow{11}{*}{$\begin{array}{l}\text { Internationaliza } \\
\text { tion }\end{array}$} & Admissions & $-.1605650^{*}$ & .0068464 & .000 & -.184861 & -.136269 \\
\hline & Academic & $-.0271680^{*}$ & .0068464 & .011 & -.051464 & -.002872 \\
\hline & Alumni & $-.0347790^{*}$ & .0068464 & .000 & -.059075 & -.010483 \\
\hline & $\begin{array}{l}\text { Placement } \\
\text { related } \\
\text { information }\end{array}$ & $-.2304260^{*}$ & .0068464 & .000 & -.254722 & -.206130 \\
\hline & $\begin{array}{l}\text { Promotion of } \\
\text { Programmes }\end{array}$ & .0086760 & .0068464 & .999 & -.015620 & .032972 \\
\hline & $\begin{array}{l}\text { B School } \\
\text { Ranking }\end{array}$ & $-.2140380^{*}$ & .0068464 & .000 & -.238334 & -.189742 \\
\hline & $\begin{array}{l}\text { Clubs and } \\
\text { Committees }\end{array}$ & $.0470760^{*}$ & .0068464 & .000 & .022780 & .071372 \\
\hline & $\begin{array}{c}\text { Academic } \\
\text { Achievement - } \\
\text { Staff }\end{array}$ & $.1073960^{*}$ & .0068464 & .000 & .083100 & .131692 \\
\hline & $\begin{array}{c}\text { Academic } \\
\text { Achievement - } \\
\text { Faculty member }\end{array}$ & $.0989590^{*}$ & .0068464 & .000 & .074663 & .123255 \\
\hline & $\begin{array}{c}\text { Academic } \\
\text { Achievement- } \\
\text { Student }\end{array}$ & $.0326890^{*}$ & .0068464 & .000 & .008393 & .056985 \\
\hline & $\begin{array}{c}\text { B School } \\
\text { Competition }\end{array}$ & $.0701260^{*}$ & .0068464 & .000 & .045830 & .094422 \\
\hline
\end{tabular}




\begin{tabular}{|c|c|c|c|c|c|c|}
\hline & $\begin{array}{c}\text { Campus } \\
\text { Information and } \\
\text { updates }\end{array}$ & $.0487420^{*}$ & .0068464 & .000 & .024446 & .073038 \\
\hline & $\begin{array}{c}\text { Case } \\
\text { Competition by } \\
\text { B School }\end{array}$ & $.0733790^{*}$ & .0068464 & .000 & 年. 049083 & .097675 \\
\hline & $\begin{array}{c}\text { Case } \\
\text { Competition by } \\
\text { Corporate }\end{array}$ & $.1106600^{*}$ & .0068464 & .000 & .086364 & .134956 \\
\hline & $\begin{array}{l}\text { Entertainment } \\
\text { and Events }\end{array}$ & $.0601990^{*}$ & .0068464 & .000 & .035903 & .084495 \\
\hline & Innovation & $-.0611570^{*}$ & .0068464 & .000 & -.085453 & -.036861 \\
\hline & $\begin{array}{l}\text { News related to } \\
\text { Education }\end{array}$ & $.1121080^{*}$ & .0068464 & .000 & .087812 & .136404 \\
\hline & $\begin{array}{l}\text { Research Related } \\
\text { Information }\end{array}$ & $-.0303760^{*}$ & .0068464 & .002 & -.054672 & -.006080 \\
\hline & $\begin{array}{c}\text { Startups } \\
\text { Information }\end{array}$ & $.0682240^{*}$ & .0068464 & .000 & .043928 & .092520 \\
\hline \multirow{14}{*}{$\begin{array}{l}\text { News related to } \\
\text { Education }\end{array}$} & Admissions & $-.2726730^{*}$ & .0068464 & .000 & -.296969 & -.248377 \\
\hline & Academic & $-.1392760^{*}$ & .0068464 & .000 & -.163572 & -.114980 \\
\hline & Alumni & $-.1468870^{*}$ & .0068464 & .000 & -.171183 & -.122591 \\
\hline & $\begin{array}{l}\text { Placement } \\
\text { related } \\
\text { information }\end{array}$ & $-.3425340^{*}$ & .0068464 & .000 & -.366830 & -.318238 \\
\hline & $\begin{array}{l}\text { Promotion of } \\
\text { Programmes }\end{array}$ & $-.1034320^{*}$ & .0068464 & .000 & -.127728 & -.079136 \\
\hline & $\begin{array}{l}\text { B School } \\
\text { Ranking }\end{array}$ & $-.3261460^{*}$ & .0068464 & .000 & -.350442 & -.301850 \\
\hline & $\begin{array}{l}\text { Clubs and } \\
\text { Committees }\end{array}$ & $-.0650320^{*}$ & .0068464 & .000 & -.089328 & -.040736 \\
\hline & $\begin{array}{c}\text { Academic } \\
\text { Achievement - } \\
\text { Staff }\end{array}$ & -.0047120 & .0068464 & 1.000 & -.029008 & .019584 \\
\hline & $\begin{array}{c}\text { Academic } \\
\text { Achievement - } \\
\text { Faculty member }\end{array}$ & -.0131490 & .0068464 & .931 & -.037445 & .011147 \\
\hline & $\begin{array}{c}\text { Academic } \\
\text { Achievement- } \\
\text { Student }\end{array}$ & $-.0794190^{*}$ & .0068464 & .000 & -.103715 & -.055123 \\
\hline & $\begin{array}{c}\text { B School } \\
\text { Competition }\end{array}$ & $-.0419820^{*}$ & .0068464 & .000 & -.066278 & -.017686 \\
\hline & $\begin{array}{c}\text { Campus } \\
\text { Information and } \\
\text { updates }\end{array}$ & $-.0633660^{*}$ & .0068464 & .000 & -.087662 & -.039070 \\
\hline & $\begin{array}{c}\text { Case } \\
\text { Competition by } \\
\text { B School }\end{array}$ & $-.0387290^{*}$ & .0068464 & .000 & -.063025 & -.014433 \\
\hline & $\begin{array}{c}\text { Case } \\
\text { Competition by }\end{array}$ & -.0014480 & .0068464 & 1.000 & -.025744 & .022848 \\
\hline
\end{tabular}




\begin{tabular}{|c|c|c|c|c|c|c|}
\hline & Corporate & & & & & \\
\hline & $\begin{array}{l}\text { Entertainment } \\
\text { and Events }\end{array}$ & $-.0519090^{*}$ & .0068464 & .000 & -.076205 & -.027613 \\
\hline & Innovation & $-.1732650^{*}$ & .0068464 & .000 & -.197561 & -.148969 \\
\hline & $\begin{array}{l}\text { Internationalizati } \\
\text { on }\end{array}$ & $-.1121080^{*}$ & .0068464 & .000 & -.136404 & -.087812 \\
\hline & $\begin{array}{l}\text { Research Related } \\
\text { Information }\end{array}$ & $-.1424840^{*}$ & .0068464 & .000 & -.166780 & -.118188 \\
\hline & $\begin{array}{c}\text { Startups } \\
\text { Information }\end{array}$ & $-.0438840^{*}$ & .0068464 & .000 & -.068180 & -.019588 \\
\hline Research & Admissions & $-.1301890^{*}$ & .0068464 & .000 & -.154485 & -.105893 \\
\hline Related & Academic & .0032080 & .0068464 & 1.000 & -.021088 & .027504 \\
\hline & Alumni & -.0044030 & .0068464 & 1.000 & -.028699 & .019893 \\
\hline & $\begin{array}{l}\text { Placement } \\
\text { related } \\
\text { information }\end{array}$ & $-.2000500^{*}$ & .0068464 & .000 & -.224346 & -.175754 \\
\hline & $\begin{array}{l}\text { Promotion of } \\
\text { Programmes }\end{array}$ & $.0390520^{*}$ & .0068464 & .000 & .014756 & .063348 \\
\hline & $\begin{array}{l}\text { B School } \\
\text { Ranking }\end{array}$ & $-.1836620^{*}$ & .0068464 & .000 & -.207958 & -.159366 \\
\hline & $\begin{array}{l}\text { Clubs and } \\
\text { Committees }\end{array}$ & $.0774520^{*}$ & .0068464 & .000 & .053156 & .101748 \\
\hline & $\begin{array}{c}\text { Academic } \\
\text { Achievement - } \\
\text { Staff }\end{array}$ & $.1377720^{*}$ & .0068464 & .000 & .113476 & .162068 \\
\hline & $\begin{array}{c}\text { Academic } \\
\text { Achievement - } \\
\text { Faculty member }\end{array}$ & $.1293350^{*}$ & .0068464 & .000 & .105039 & .153631 \\
\hline & $\begin{array}{c}\text { Academic } \\
\text { Achievement- } \\
\text { Student }\end{array}$ & $.0630650^{*}$ & .0068464 & .000 & .038769 & .087361 \\
\hline & $\begin{array}{c}\text { B School } \\
\text { Competition }\end{array}$ & $.1005020^{*}$ & .0068464 & .000 & .076206 & .124798 \\
\hline & $\begin{array}{l}\text { Campus } \\
\text { Information and } \\
\text { updates }\end{array}$ & $.0791180^{*}$ & .0068464 & .000 & .054822 & .103414 \\
\hline & $\begin{array}{c}\text { Case } \\
\text { Competition by } \\
\text { B School }\end{array}$ & $.1037550^{*}$ & .0068464 & .000 & .079459 & .128051 \\
\hline & $\begin{array}{c}\text { Case } \\
\text { Competition by } \\
\text { Corporate }\end{array}$ & $.1410360^{*}$ & .0068464 & .000 & .116740 & .165332 \\
\hline & $\begin{array}{l}\text { Entertainment } \\
\text { and Events }\end{array}$ & $.0905750^{*}$ & .0068464 & .000 & .066279 & .114871 \\
\hline & Innovation & $-.0307810^{*}$ & .0068464 & .001 & -.055077 & -.006485 \\
\hline & $\begin{array}{c}\text { Internationalizati } \\
\text { on }\end{array}$ & $.0303760^{*}$ & .0068464 & .002 & .006080 & .054672 \\
\hline
\end{tabular}




\begin{tabular}{|c|c|c|c|c|c|c|}
\hline & $\begin{array}{l}\text { News related to } \\
\text { Education }\end{array}$ & $.1424840^{*}$ & .0068464 & .000 & .118188 & .166780 \\
\hline & $\begin{array}{l}\text { Startups } \\
\text { Information }\end{array}$ & $.0986000^{*}$ & .0068464 & .000 & .074304 & .122896 \\
\hline \multirow{19}{*}{$\begin{array}{l}\text { Startups } \\
\text { Information }\end{array}$} & Admissions & $-.2287890^{*}$ & .0068464 & .000 & -.253085 & -.204493 \\
\hline & Academic & $-.0953920^{*}$ & .0068464 & .000 & -.119688 & -.071096 \\
\hline & Alumni & $-.1030030^{*}$ & .0068464 & .000 & -.127299 & -.078707 \\
\hline & $\begin{array}{l}\text { Placement } \\
\text { related } \\
\text { information }\end{array}$ & $-.2986500^{*}$ & .0068464 & .000 & -.322946 & -.274354 \\
\hline & $\begin{array}{l}\text { Promotion of } \\
\text { Programmes }\end{array}$ & $-.0595480^{*}$ & .0068464 & .000 & -.083844 & -.035252 \\
\hline & $\begin{array}{l}\text { B School } \\
\text { Ranking }\end{array}$ & $-.2822620^{*}$ & .0068464 & .000 & -.306558 & -.257966 \\
\hline & $\begin{array}{l}\text { Clubs and } \\
\text { Committees }\end{array}$ & -.0211480 & .0068464 & .188 & -.045444 & .003148 \\
\hline & $\begin{array}{c}\text { Academic } \\
\text { Achievement - } \\
\text { Staff }\end{array}$ & $.0391720^{*}$ & .0068464 & .000 & .014876 & .063468 \\
\hline & $\begin{array}{c}\text { Academic } \\
\text { Achievement - } \\
\text { Faculty member }\end{array}$ & $.0307350^{*}$ & .0068464 & .001 & .006439 & .055031 \\
\hline & $\begin{array}{c}\text { Academic } \\
\text { Achievement- } \\
\text { Student }\end{array}$ & $-.0355350^{*}$ & .0068464 & .000 & -.059831 & -.011239 \\
\hline & $\begin{array}{c}\text { B School } \\
\text { Competition }\end{array}$ & .0019020 & .0068464 & 1.000 & -.022394 & .026198 \\
\hline & $\begin{array}{c}\text { Campus } \\
\text { Information and } \\
\text { updates }\end{array}$ & -.0194820 & .0068464 & .326 & -.043778 & .004814 \\
\hline & $\begin{array}{c}\text { Case } \\
\text { Competition by } \\
\text { B School }\end{array}$ & .0051550 & .0068464 & 1.000 & -.019141 & .029451 \\
\hline & $\begin{array}{c}\text { Case } \\
\text { Competition by } \\
\text { Corporate }\end{array}$ & $.0424360^{*}$ & .0068464 & .000 & .018140 & .066732 \\
\hline & $\begin{array}{l}\text { Entertainment } \\
\text { and Events }\end{array}$ & -.0080250 & .0068464 & 1.000 & -.032321 & .016271 \\
\hline & Innovation & $-.1293810^{*}$ & .0068464 & .000 & -.153677 & -.105085 \\
\hline & $\begin{array}{l}\text { Internationalizati } \\
\text { on }\end{array}$ & $-.0682240^{*}$ & .0068464 & .000 & -.092520 & -.043928 \\
\hline & $\begin{array}{l}\text { News related to } \\
\text { Education }\end{array}$ & $.0438840^{*}$ & .0068464 & .000 & .019588 & .068180 \\
\hline & $\begin{array}{l}\text { Research Related } \\
\text { Information }\end{array}$ & $-.0986000^{*}$ & .0068464 & .000 & -.122896 & -.074304 \\
\hline
\end{tabular}

*. The mean difference is significant at the 0.05 level. 
Table 5. No significant difference in click through ratio for advertisement - category

\begin{tabular}{|c|c|}
\hline Categories & $\begin{array}{l}\text { Statistically NO significant difference in click } \\
\text { through ratio in advertisement in the following } \\
\text { categories }\end{array}$ \\
\hline Admissions & NIL \\
\hline \multirow[t]{2}{*}{ Academic } & Alumni $(\mathrm{p}=1.000)$ \\
\hline & Research Related Information ( $\mathrm{p}=1.000)$ \\
\hline \multirow[t]{2}{*}{ Alumni } & Academic $(\mathrm{p}=1.000)$ \\
\hline & Research Related Information $(\mathrm{p}=1.000)$ \\
\hline Placement related information & B School Ranking $(\mathrm{p}=0.666)$ \\
\hline \multirow[t]{2}{*}{ Promotion of Programmes } & Academic Achievement - Student $(\mathrm{p}=0.057)$ \\
\hline & Internationalization $(\mathrm{p}=0.999)$ \\
\hline B School Ranking & Placement related information $(\mathrm{p}=0.666)$ \\
\hline \multirow[t]{5}{*}{ Clubs and Committees } & Academic Achievement- Student $(\mathrm{p}=0.856)$ \\
\hline & B School Competition $(\mathrm{p}=0.088)$ \\
\hline & Campus Information and updates $(\mathrm{p}=1.000)$ \\
\hline & Entertainment and Events $(\mathrm{p}=0.933)$ \\
\hline & Startups Information $(\mathrm{p}=0.188)$ \\
\hline \multirow[t]{3}{*}{ Academic Achievement - Staff } & Academic Achievement - Faculty member $(\mathrm{p}=1.000)$ \\
\hline & Case Competition by Corporate $(\mathrm{p}=0.978)$ \\
\hline & News related to Education $(\mathrm{p}=0.931)$ \\
\hline \multirow[t]{3}{*}{ Academic Achievement - Faculty member } & Academic Achievement - Staff $(\mathrm{p}=1.000)$ \\
\hline & Case Competition by Corporate $(\mathrm{p}=0.978)$ \\
\hline & News related to Education $(\mathrm{p}=0.931)$ \\
\hline \multirow[t]{3}{*}{ Academic Achievement- Student } & Promotion of Programmes $(\mathrm{p}=0.057)$ \\
\hline & Clubs and Committees $(\mathrm{p}=0.856)$ \\
\hline & Campus Information and updates $(\mathrm{p}=0.703)$ \\
\hline \multirow[t]{5}{*}{ B School Competition } & Clubs and Committees $(\mathrm{p}=0.088)$ \\
\hline & Campus Information and updates $(\mathrm{p}=0.172)$ \\
\hline & Case Competition by B School (p=1.000) \\
\hline & Entertainment and Events $(\mathrm{p}=0.997)$ \\
\hline & Startups Information $(\mathrm{p}=1.000)$ \\
\hline \multirow[t]{5}{*}{ Campus Information and updates } & Clubs and Committees $(\mathrm{p}=1.000)$ \\
\hline & Academic Achievement - Student $(\mathrm{p}=0.703)$ \\
\hline & B School Competition $(\mathrm{p}=0.172)$ \\
\hline & Entertainment and Events $(\mathrm{p}=0.982)$ \\
\hline & Startups Information $(\mathrm{p}=0.326)$ \\
\hline \multirow[t]{3}{*}{ Case Competition by B School } & B School Competition $(\mathrm{p}=1.000)$ \\
\hline & Entertainment and Events $(\mathrm{p}=0.930)$ \\
\hline & Startups Information $(\mathrm{p}=1.000)$ \\
\hline \multirow[t]{3}{*}{ Case Competition by Corporate } & Academic Achievement - Staff $(\mathrm{p}=1.000)$ \\
\hline & Academic Achievement - Faculty member $(\mathrm{p}=0.978)$ \\
\hline & News related to Education $(\mathrm{p}=1.000)$ \\
\hline Entertainment and Events & Clubs and Committees $(\mathrm{p}=0.933)$ \\
\hline
\end{tabular}


B School Competition $(\mathrm{p}=0.997)$

Campus Information and updates $(\mathrm{p}=0.982)$

Case Competition by B School $(\mathrm{p}=0.930)$

Startups Information $(\mathrm{p}=1.000)$

Innovation

Internationalization

News related to

Education

Research Related Information

Startups Information
NIL

Promotion of Programmes $(\mathrm{p}=0.999)$

Academic Achievement - Staff $(\mathrm{p}=1.000)$

Academic Achievement - Faculty member $(\mathrm{p}=0.931)$

Case Competition by Corporate $(\mathrm{p}=1.000)$ Academic $(\mathrm{p}=1.000)$

Alumni $(\mathrm{p}=1.000)$

Clubs and Committees $(\mathrm{p}=0.188)$

B School Competition $(\mathrm{p}=1.000)$

Campus Information and updates $(\mathrm{p}=0.326)$

Case Competition by B School $(\mathrm{p}=1.000)$

Entertainment and Events $(\mathrm{p}=1.000)$

Table 6. Intensity index of categories of advertisement

Category

Placement related information

B School Ranking

Admissions

Innovation

Alumni

Research Related Information

Academic

Internationalization

Promotion of Programmes

Academic Achievement-Student

Clubs and Committees

Campus Information and updates

Entertainment and Events
100

100

100

100

100

100

100

100

100

100

100

100

100
Mean

388585

.372197

.318724

.219316

192938

188535

.185327

.158159

.149483

.125470

.0302948

0578416

.0381551

.0307868

.1286860

.0184693

.0360562

.0094075

0578416
0381551
0307868

.0273953

.111083

.109417

.0121003

.097960

.0407794 
Startups Information

B School Competition

Case Competition by B School

Academic Achievement - Faculty member
100

100

100

100

100

100

100
Academic Achievement - Staff

Case Competition by Corporate
News related to Education
.089935

.088033

.084780

.059200

.0199321

.0205245

.0226693

.0200967

.0166172

.0129860

.047499

.0129860

.0178411

Table No 6 indicates the intensity index of the category of advertisement. It shows that placement related information $(\mathrm{M}=.388585)$, B school ranking $(\mathrm{M}=.372197)$ and admission $(\mathrm{M}=.318724)$ related advertisement have highest click though ratio while Case Competition by B School $(\mathrm{M}=.084780)$, Academic Achievement - Faculty member $(M=.059200)$ and Academic Achievement - Staff $(M=.050763)$ have lower click though ratio. It can be concluded that advertisement related to placement information, B school ranking and admission related have highest click though ratio while Case Competition by B School, Academic Achievement - Faculty member and Academic Achievement - Staff have lower click though ratio.

Table 7. Descriptive information of format type of advertisement

\begin{tabular}{|c|c|c|c|c|c|c|c|c|}
\hline \multirow{2}{*}{$\begin{array}{c}\text { Format click } \\
\text { through } \\
\text { ratio }\end{array}$} & \multirow{2}{*}{$\mathbf{N}$} & \multirow{2}{*}{ Mean } & \multirow{2}{*}{$\begin{array}{c}\text { Std. } \\
\text { Deviatio } \\
\quad n\end{array}$} & \multirow{2}{*}{$\begin{array}{l}\text { Std. } \\
\text { Error }\end{array}$} & \multicolumn{2}{|c|}{$\begin{array}{l}\text { 95\% Confidence } \\
\text { Interval for Mean }\end{array}$} & \multirow{2}{*}{$\begin{array}{l}\text { Minim } \\
\text { um }\end{array}$} & \multirow{2}{*}{$\begin{array}{c}\text { Maxim } \\
\text { um }\end{array}$} \\
\hline & & & & & $\begin{array}{l}\text { Lower } \\
\text { Bound }\end{array}$ & $\begin{array}{l}\text { Upper } \\
\text { Bound }\end{array}$ & & \\
\hline Text & 100 & .527804 & .0219978 & .0021998 & .523439 & .532169 & .4404 & .5463 \\
\hline $\begin{array}{c}\text { Carousel } \\
\text { banner }\end{array}$ & 100 & .189540 & .0380747 & .0038075 & .181985 & .197095 & .1235 & .2637 \\
\hline $\begin{array}{c}\text { HTML five } \\
\text { advertisem } \\
\text { ent }\end{array}$ & 100 & .319876 & .0129804 & .0012980 & .317300 & .322452 & .3000 & .3442 \\
\hline $\begin{array}{l}\text { Interactive } \\
\text { video }\end{array}$ & 100 & .484586 & .0219200 & .0021920 & .480237 & .488935 & .4649 & .5701 \\
\hline Short video & 100 & .416556 & .0271736 & .0027174 & .411164 & .421948 & .3895 & .5228 \\
\hline Long Video & 100 & .317296 & .0380778 & .0038078 & .309741 & .324851 & .2757 & .4500 \\
\hline $\begin{array}{l}\text { Desktop } \\
\text { banner }\end{array}$ & 100 & .118469 & .0284388 & .0028439 & .112826 & .124112 & .0050 & .1526 \\
\hline $\begin{array}{l}\text { Mobile } \\
\text { banner }\end{array}$ & 100 & .280058 & 0399739 & .0039974 & .272126 & .287990 & .1045 & .3143 \\
\hline $\begin{array}{l}\text { Dynamic } \\
\text { text }\end{array}$ & 100 & .538787 & .0268667 & .0026867 & .533456 & .544118 & .4418 & .5730 \\
\hline $\begin{array}{l}\text { Text and } \\
\text { phone no }\end{array}$ & 100 & .482279 & .0195872 & .0019587 & .478392 & .486166 & .3940 & .4996 \\
\hline
\end{tabular}




\begin{tabular}{ccccccccc}
\hline $\begin{array}{c}\text { Dynamic } \\
\text { banner }\end{array}$ & 100 & .206650 & .0258542 & .0025854 & .201520 & .211780 & .0955 & .2345 \\
Extension & 100 & .618782 & .0385859 & .0038586 & .611126 & .626438 & .4502 & .6541 \\
Total & 1200 & .375057 & .1552832 & .0044826 & .366262 & .383852 & .0050 & .6541 \\
\hline
\end{tabular}

The hypothesis (B) Ho: There is no significant difference in the click through ratio for the format of the advertisement which was crafted to check if there was any significant difference in the click through ratio and its relation with the format of the advertisement. The information given in Table 7 gives descriptive statistical data, including mean, standard deviation and $95 \%$ confidence intervals for the dependent variable- click through score- for each of the format of the advertisement on Google where all the twelve categories have been listed.

Table 7 is the output of the ANOVA analysis which is used to test the Hypothesis (B) to understand if there is a statistically significant difference between the group means of click through ratio and the Format of the advertisement. It can be inferred from the significance value 0.000 (i.e., $\mathrm{p}=0.000$ ), which is below 0.05 , that there is a statistically significant difference between the click through ratio and the format of the Advertisement, which clearly indicates that different advertisement under different formats have a different click through ratios. To know which of those specific formats differed in the click ratio, Tukey post hoc test was used and Table 7 gives the output which includes multiple comparisons for the format type. From the multiple comparisons Table 7 it can be inferred that there is a statistically significant difference in the click through ratio and the advertisement that were in Text format in comparison with all other categories $(p=0.000)$, but there is no significant difference between the advertisement of Text format and the advertisement of under Dynamic text $(\mathrm{p}=0.262)$. For the advertisement of the format 'Carousel banner', 'Short video', 'Desktop banner', 'Mobile banner', 'Dynamic banner' and 'Extension' it can be statistically inferred that there is a significant difference in the click through ratio of the advertisement in comparison with all other formats $(\mathrm{p}=0.000)$. Table 7 gives the details for all the formats for which there is no statistical significant difference in the click through ratio of the advertisement when compared to other formats.

Table 8. ANOVA analysis - format type

Format type click through ratio

\begin{tabular}{cccccc}
\hline & $\begin{array}{c}\text { Sum of } \\
\text { Squares }\end{array}$ & df & $\begin{array}{c}\text { Mean } \\
\text { Square }\end{array}$ & F & Sig. \\
& 27.878 & 11 & 2.534 & 2912.935 & .000 \\
Between Groups & 1.034 & 1188 & .001 & & \\
Within Groups & 28.911 & 1199 & & & \\
Total & & &
\end{tabular}

Table 9. Multiple comparisons of format type

\begin{tabular}{|c|c|c|c|c|c|c|}
\hline \multicolumn{7}{|c|}{$\begin{array}{l}\text { Dependent Variable: Format type click through ratio } \\
\text { Tukey HSD }\end{array}$} \\
\hline (I) Format & (J) Format & $\begin{array}{c}\text { Mean } \\
\text { Difference } \\
(\mathbf{I}-\mathbf{J})\end{array}$ & Std. Error & Sig. & $\begin{array}{l}\text { Lower } \\
\text { Bound }\end{array}$ & $\begin{array}{l}\text { Upper } \\
\text { Bound }\end{array}$ \\
\hline \multirow[t]{10}{*}{ Text } & Carousel banner & $.3382640^{*}$ & .0041714 & .000 & .324604 & .351924 \\
\hline & HTML five & $.2079280^{*}$ & .0041714 & .000 & .194268 & .221588 \\
\hline & $\begin{array}{l}\text { advertisement } \\
\text { Interactive video }\end{array}$ & $.0432180^{*}$ & .0041714 & .000 & .029558 & .056878 \\
\hline & Short video & $.1112480^{*}$ & .0041714 & .000 & .097588 & .124908 \\
\hline & Long Video & $.2105080^{*}$ & .0041714 & .000 & .196848 & .224168 \\
\hline & Desktop banner & $.4093350^{*}$ & .0041714 & .000 & .395675 & .422995 \\
\hline & Mobile banner & $.2477460^{*}$ & .0041714 & .000 & .234086 & .261406 \\
\hline & Dynamic text & -.0109830 & .0041714 & .262 & -.024643 & .002677 \\
\hline & Text and phone no & $.0455250^{*}$ & .0041714 & .000 & .031865 & .059185 \\
\hline & Dynamic banner & $.3211540^{*}$ & .0041714 & .000 & .307494 & .334814 \\
\hline
\end{tabular}




\begin{tabular}{|c|c|c|c|c|c|c|}
\hline \multirow{3}{*}{$\begin{array}{l}\text { Carousel } \\
\text { banner }\end{array}$} & Extension & $-.0909780^{*}$ & .0041714 & .000 & -.104638 & -.077318 \\
\hline & Text & $-.3382640^{*}$ & .0041714 & .000 & -.351924 & -.324604 \\
\hline & $\begin{array}{c}\text { HTML five } \\
\text { advertisement }\end{array}$ & $-.1303360^{*}$ & .0041714 & .000 & -.143996 & -.116676 \\
\hline \multirow{17}{*}{$\begin{array}{l}\text { HTML } \\
\text { five } \\
\text { advertise } \\
\text { ment }\end{array}$} & Interactive video & $-.2950460^{*}$ & .0041714 & .000 & -.308706 & -.281386 \\
\hline & Short video & $-.2270160^{*}$ & .0041714 & .000 & -.240676 & -.213356 \\
\hline & Long Video & $-.1277560^{*}$ & .0041714 & .000 & -.141416 & -.114096 \\
\hline & Desktop banner & $.0710710^{*}$ & .0041714 & .000 & .057411 & .084731 \\
\hline & Mobile banner & $-.0905180^{*}$ & .0041714 & .000 & -.104178 & -.076858 \\
\hline & Dynamic text & $-.3492470^{*}$ & .0041714 & .000 & -.362907 & -.335587 \\
\hline & Text and phone no & $-.2927390^{*}$ & .0041714 & .000 & -.306399 & -.279079 \\
\hline & Dynamic banner & $-.0171100^{*}$ & .0041714 & .003 & -.030770 & -.003450 \\
\hline & Extension & $-.4292420^{*}$ & .0041714 & .000 & -.442902 & -.415582 \\
\hline & Text & $-.2079280^{*}$ & .0041714 & .000 & -.221588 & -.194268 \\
\hline & Carousel banner & $.1303360^{*}$ & .0041714 & .000 & .116676 & .143996 \\
\hline & Interactive video & $-.1647100^{*}$ & .0041714 & .000 & -.178370 & -.151050 \\
\hline & Short video & $-.0966800^{*}$ & .0041714 & .000 & -.110340 & -.083020 \\
\hline & Long Video & .0025800 & .0041714 & 1.000 & -.011080 & .016240 \\
\hline & Desktop banner & $.2014070^{*}$ & .0041714 & .000 & .187747 & .215067 \\
\hline & Mobile banner & $.0398180^{*}$ & .0041714 & .000 & .026158 & .053478 \\
\hline & Dynamic text & $-.2189110^{*}$ & .0041714 & .000 & -.232571 & -.205251 \\
\hline \multirow{7}{*}{$\begin{array}{l}\text { Interactiv } \\
\text { e video }\end{array}$} & Text and phone no & $-.1624030^{*}$ & .0041714 & .000 & -.176063 & -.148743 \\
\hline & Dynamic banner & $.1132260^{*}$ & .0041714 & .000 & .099566 & .126886 \\
\hline & Extension & $-.2989060^{*}$ & .0041714 & .000 & -.312566 & -.285246 \\
\hline & Text & $-.0432180^{*}$ & .0041714 & .000 & -.056878 & -.029558 \\
\hline & Carousel banner & $.2950460^{*}$ & .0041714 & .000 & .281386 & .308706 \\
\hline & $\begin{array}{c}\text { HTML five } \\
\text { advertisement }\end{array}$ & $.1647100^{*}$ & .0041714 & .000 & .151050 & .178370 \\
\hline & Short video & $.0680300^{*}$ & .0041714 & .000 & .054370 & .081690 \\
\hline \multirow{14}{*}{$\begin{array}{l}\text { Short } \\
\text { video }\end{array}$} & Long Video & $.1672900^{*}$ & .0041714 & .000 & .153630 & .180950 \\
\hline & Desktop banner & $.3661170^{*}$ & .0041714 & .000 & .352457 & .379777 \\
\hline & Mobile banner & $.2045280^{*}$ & .0041714 & .000 & .190868 & .218188 \\
\hline & Dynamic text & $-.0542010^{*}$ & .0041714 & .000 & -.067861 & -.040541 \\
\hline & Text and phone no & .0023070 & .0041714 & 1.000 & -.011353 & .015967 \\
\hline & Dynamic banner & $.2779360^{*}$ & .0041714 & .000 & .264276 & .291596 \\
\hline & Extension & $-.1341960^{*}$ & .0041714 & .000 & -.147856 & -.120536 \\
\hline & Text & $-.1112480^{*}$ & .0041714 & .000 & -.124908 & -.097588 \\
\hline & Carousel banner & $.2270160^{*}$ & .0041714 & .000 & .213356 & .240676 \\
\hline & $\begin{array}{c}\text { HTML five } \\
\text { advertisement }\end{array}$ & $.0966800^{*}$ & .0041714 & .000 & .083020 & .110340 \\
\hline & Interactive video & $-.0680300^{*}$ & .0041714 & .000 & -.081690 & -.054370 \\
\hline & Long Video & $.0992600^{*}$ & .0041714 & .000 & .085600 & .112920 \\
\hline & Desktop banner & $.2980870^{*}$ & .0041714 & .000 & .284427 & .311747 \\
\hline & Mobile banner & $.1364980^{*}$ & .0041714 & .000 & .122838 & .150158 \\
\hline
\end{tabular}




\begin{tabular}{|c|c|c|c|c|c|c|}
\hline \multirow{12}{*}{$\begin{array}{l}\text { Long } \\
\text { Video }\end{array}$} & Dynamic text & $-.1222310^{*}$ & .0041714 & .000 & -.135891 & -.108571 \\
\hline & Text and phone no & $-.0657230^{*}$ & .0041714 & .000 & -.079383 & -.052063 \\
\hline & Dynamic banner & $.2099060^{*}$ & .0041714 & .000 & .196246 & .223566 \\
\hline & Extension & $-.2022260^{*}$ & .0041714 & .000 & -.215886 & -.188566 \\
\hline & Text & $-.2105080^{*}$ & .0041714 & .000 & -.224168 & -.196848 \\
\hline & Carousel banner & $.1277560^{*}$ & .0041714 & .000 & .114096 & .141416 \\
\hline & $\begin{array}{l}\text { HTML five } \\
\text { advertisement }\end{array}$ & -.0025800 & .0041714 & 1.000 & -.016240 & .011080 \\
\hline & Interactive video & $-.1672900^{*}$ & .0041714 & .000 & -.180950 & -.153630 \\
\hline & Short video & $-.0992600^{*}$ & .0041714 & .000 & -.112920 & -.085600 \\
\hline & Desktop banner & $.1988270^{*}$ & .0041714 & .000 & .185167 & .212487 \\
\hline & Mobile banner & $.0372380^{*}$ & .0041714 & .000 & .023578 & .050898 \\
\hline & Dynamic text & $-.2214910^{*}$ & .0041714 & .000 & -.235151 & -.207831 \\
\hline \multirow{7}{*}{$\begin{array}{l}\text { Desktop } \\
\text { banner }\end{array}$} & Text and phone no & $-.1649830^{*}$ & .0041714 & .000 & -.178643 & -.151323 \\
\hline & Dynamic banner & $.1106460^{*}$ & .0041714 & .000 & .096986 & .124306 \\
\hline & Extension & $-.3014860^{*}$ & .0041714 & .000 & -.315146 & -.287826 \\
\hline & Text & $-.4093350^{*}$ & .0041714 & .000 & -.422995 & -.395675 \\
\hline & Carousel banner & $-.0710710^{*}$ & .0041714 & .000 & -.084731 & -.057411 \\
\hline & $\begin{array}{c}\text { HTML five } \\
\text { advertisement }\end{array}$ & $-.2014070^{*}$ & .0041714 & .000 & -.215067 & -.187747 \\
\hline & Interactive video & $-.3661170^{*}$ & .0041714 & .000 & -.379777 & -.352457 \\
\hline \multirow{15}{*}{$\begin{array}{l}\text { Mobile } \\
\text { banner }\end{array}$} & Short video & $-.2980870^{*}$ & .0041714 & .000 & -.311747 & -.284427 \\
\hline & Long Video & $-.1988270^{*}$ & .0041714 & .000 & -.212487 & -.185167 \\
\hline & Mobile banner & $-.1615890^{*}$ & .0041714 & .000 & -.175249 & -.147929 \\
\hline & Dynamic text & $-.4203180^{*}$ & .0041714 & .000 & -.433978 & -.406658 \\
\hline & Text and phone no & $-.3638100^{*}$ & .0041714 & .000 & -.377470 & -.350150 \\
\hline & Dynamic banner & $-.0881810^{*}$ & .0041714 & .000 & -.101841 & -.074521 \\
\hline & Extension & $-.5003130^{*}$ & .0041714 & .000 & -.513973 & -.486653 \\
\hline & Text & $-.2477460^{*}$ & .0041714 & .000 & -.261406 & -.234086 \\
\hline & Carousel banner & $.0905180^{*}$ & .0041714 & .000 & .076858 & .104178 \\
\hline & $\begin{array}{c}\text { HTML five } \\
\text { advertisement }\end{array}$ & $-.0398180^{*}$ & .0041714 & .000 & -.053478 & -.026158 \\
\hline & Interactive video & $-.2045280^{*}$ & .0041714 & .000 & -.218188 & -.190868 \\
\hline & Short video & $-.1364980^{*}$ & .0041714 & .000 & -.150158 & -.122838 \\
\hline & Long Video & $-.0372380^{*}$ & .0041714 & .000 & -.050898 & -.023578 \\
\hline & Desktop banner & $.1615890^{*}$ & .0041714 & .000 & .147929 & .175249 \\
\hline & Dynamic text & $-.2587290^{*}$ & .0041714 & .000 & -.272389 & -.245069 \\
\hline \multirow{7}{*}{$\begin{array}{l}\text { Dynamic } \\
\text { text }\end{array}$} & Text and phone no & $-.2022210^{*}$ & .0041714 & .000 & -.215881 & -.188561 \\
\hline & Dynamic banner & $.0734080^{*}$ & .0041714 & .000 & .059748 & .087068 \\
\hline & Extension & $-.3387240^{*}$ & .0041714 & .000 & -.352384 & -.325064 \\
\hline & Text & .0109830 & .0041714 & .262 & -.002677 & .024643 \\
\hline & Carousel banner & $.3492470^{*}$ & .0041714 & .000 & .335587 & .362907 \\
\hline & $\begin{array}{l}\text { HTML five } \\
\text { advertisement }\end{array}$ & $.2189110^{*}$ & .0041714 & .000 & .205251 & .232571 \\
\hline & Interactive video & $.0542010^{*}$ & .0041714 & .000 & .040541 & .067861 \\
\hline
\end{tabular}




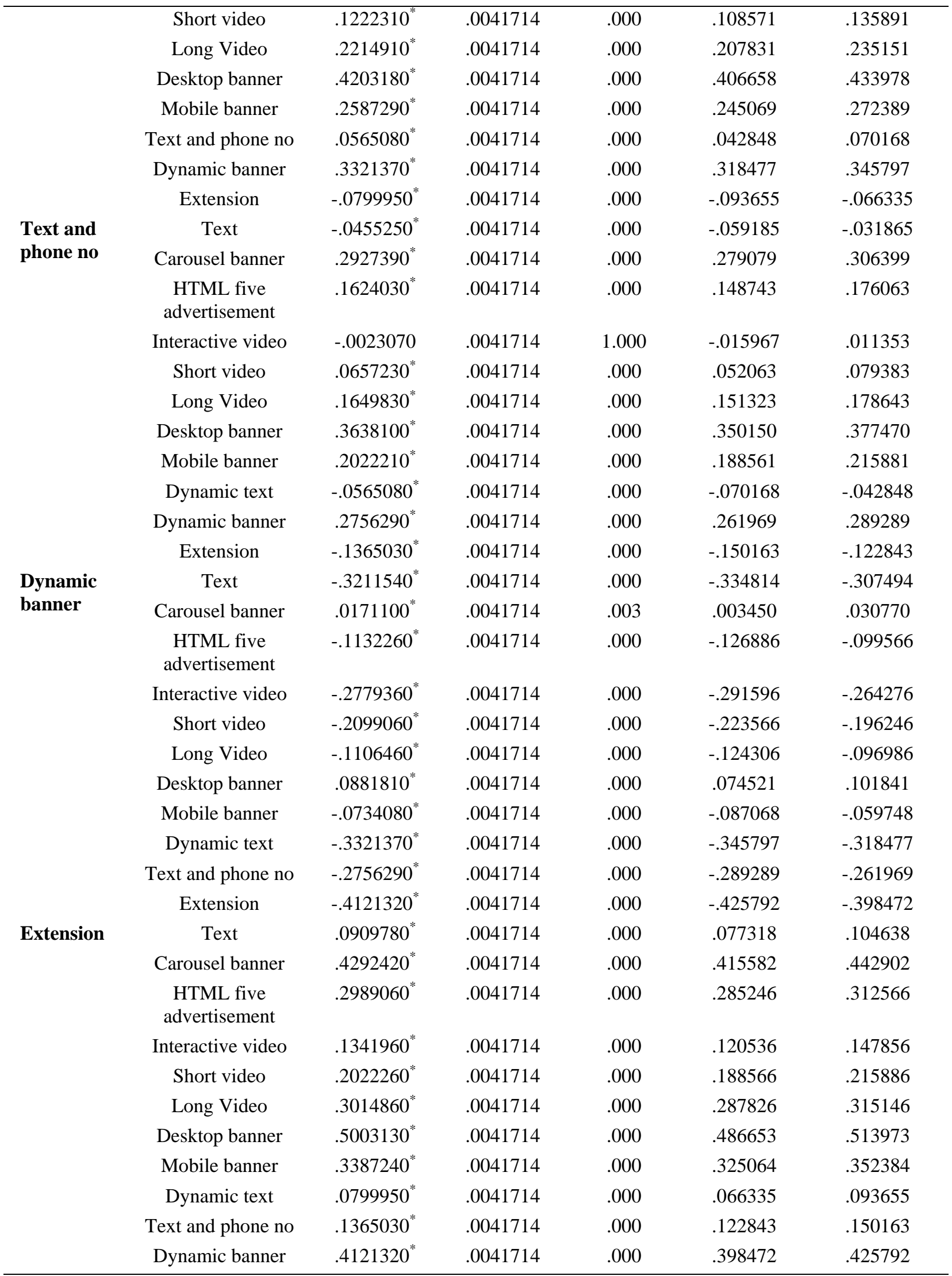

*. The mean difference is significant at the 0.05 level. 
Table 10. No significant difference in click through ratio for advertisement - FORMAT TYPE

\begin{tabular}{ll}
\hline Format Type & $\begin{array}{l}\text { Statistically no significant difference in click } \\
\text { through ratio in advertisement posted in the } \\
\text { following format }\end{array}$ \\
\hline Text & Dynamic text $(\mathrm{p}=0.262)$ \\
Carousel banner & NIL \\
HTML five advertisement & Long Video $(\mathrm{p}=1.000)$ \\
Interactive video & Text and phone no $(\mathrm{p}=1.000)$ \\
Short video & NIL \\
Long Video & HTML five advertisement $(\mathrm{p}=1.000)$ \\
Desktop banner & NIL \\
Mobile banner & NIL \\
Dynamic text & Text $(\mathrm{p}=0.262)$ \\
Text and phone no & Interactive video $(\mathrm{p}=1.000)$ \\
Dynamic banner & NIL \\
Extension & NIL \\
\hline
\end{tabular}

\begin{tabular}{cccc}
\hline Table 11. Intensity index of format type- click through ratio & & & \\
\hline & $\mathbf{N}$ & Mean & Std. Deviation \\
\hline Extension & 100 & .618782 & .0385859 \\
Dynamic text & 100 & .538787 & .0268667 \\
Text & 100 & .527804 & .0219978 \\
Interactive video & 100 & .484586 & .0219200 \\
Text and phone no & 100 & .482279 & .0195872 \\
Short video & 100 & .416556 & .0271736 \\
HTML five advertisement & 100 & .319876 & .0129804 \\
Long Video & 100 & .317296 & .0380778 \\
Mobile banner & 100 & .280058 & .0399739 \\
Dynamic banner & 100 & .206650 & .0258542 \\
Carousel banner & 100 & .189540 & .0380747 \\
Desktop banner & 100 & .118469 & .0284388
\end{tabular}

Table 11 indicates the intensity index of the format type of advertisement. It shows that extension $(\mathrm{M}=.618782)$, dynamic text $(\mathrm{M}=.538787)$ and text $(\mathrm{M}=.527804)$ advertisement have the highest click though ratio while dynamic banner $(M=.206650)$, Carousel banner $(M=.189540)$ and Desktop banner $(M=.118469)$ have lower click though ratio. It can be concluded that advertisement related extension, dynamic text and text while dynamic banner, Carousel banner and Desktop banner have lower click though ratio. 
Table 12. Descriptive information - day and time of advertisement

\begin{tabular}{|c|c|c|c|c|c|c|c|c|}
\hline \multicolumn{9}{|c|}{ Time click through ratio } \\
\hline & \multirow[t]{2}{*}{$\mathrm{N}$} & \multirow[t]{2}{*}{ Mean } & \multirow[t]{2}{*}{$\begin{array}{c}\text { Std. } \\
\text { Deviation }\end{array}$} & \multirow[t]{2}{*}{ Std. Error } & \multicolumn{2}{|c|}{$\begin{array}{l}95 \% \text { Confidence } \\
\text { Interval for Mean }\end{array}$} & \multirow[t]{2}{*}{$\begin{array}{c}\text { Minimu } \\
\mathrm{m}\end{array}$} & \multirow[t]{2}{*}{$\begin{array}{c}\text { Maxim } \\
\text { um }\end{array}$} \\
\hline & & & & & $\begin{array}{l}\text { Lower } \\
\text { Bound }\end{array}$ & $\begin{array}{l}\text { Upper } \\
\text { Bound }\end{array}$ & & \\
\hline $\begin{array}{l}\text { Week Day } 00.00 \\
\text { am to } 08.00 \text { am }\end{array}$ & 100 & .149671 & .0093841 & .0009384 & .147809 & .151533 & .1326 & .1642 \\
\hline $\begin{array}{l}\text { Week Day } 08.01 \\
\text { am to } 10.00 \text { am }\end{array}$ & 100 & .292571 & .0043112 & .0004311 & .291716 & .293426 & .2849 & .3000 \\
\hline $\begin{array}{l}\text { Week Day } 10.01 \\
\text { am to } 05 \# 00 \mathrm{pm}\end{array}$ & 100 & .653162 & .0120479 & .0012048 & .650771 & .655553 & .6327 & .6745 \\
\hline $\begin{array}{l}\text { Week Day } 05.01 \\
\text { pm to } 08.00 \mathrm{pm}\end{array}$ & 100 & .310296 & .0014062 & .0001406 & .310017 & .310575 & .3080 & .3126 \\
\hline $\begin{array}{l}\text { Week Day } 08.01 \\
\text { pm to } 10.00 \mathrm{pm}\end{array}$ & 100 & .197765 & .0109310 & .0010931 & .195596 & .199934 & .1788 & .2147 \\
\hline $\begin{array}{l}\text { Week Day } 10.00 \\
\text { pm to } 11.59 \text { pm }\end{array}$ & 100 & .136597 & .0132028 & .0013203 & .133977 & .139217 & .1144 & .1566 \\
\hline $\begin{array}{l}\text { Week End } 00.00 \\
\text { am to } 08.00 \text { am }\end{array}$ & 100 & .052875 & .0015227 & .0001523 & .052573 & .053177 & .0497 & .0554 \\
\hline $\begin{array}{l}\text { Week End } 08.01 \\
\text { am to } 10.00 \mathrm{am}\end{array}$ & 100 & .269251 & .0115473 & .0011547 & .266960 & .271542 & .2482 & .2866 \\
\hline $\begin{array}{l}\text { Week End } 10.01 \\
\text { am to } 05 \# 00 \mathrm{pm}\end{array}$ & 100 & .400358 & .0074065 & .0007406 & .398888 & .401828 & .3878 & .4123 \\
\hline $\begin{array}{l}\text { Week End } 05.01 \\
\text { pm to } 08.00 \mathrm{pm}\end{array}$ & 100 & .244807 & .0110917 & .0011092 & .242606 & .247008 & .2267 & .2645 \\
\hline $\begin{array}{l}\text { Week End } 08.01 \\
\text { pm to } 10.00 \mathrm{pm}\end{array}$ & 100 & .105265 & .0037481 & .0003748 & .104521 & .106009 & .0988 & .1127 \\
\hline $\begin{array}{l}\text { Week End } 10.00 \\
\text { pm to } 11.59 \mathrm{pm}\end{array}$ & 100 & .066409 & .0045813 & .0004581 & .065500 & .067318 & .0577 & .0740 \\
\hline Total & 1200 & .239919 & .1607497 & .0046404 & .230815 & .249023 & .0497 & .6745 \\
\hline
\end{tabular}

The hypothesis $(C)$ Ho: There is no significant difference in the click through ratio for the day and time of the advertisement was crafted to check if there was any significant difference in the click through ratio and its relation to the weekday/weekend and the timing of the advertisement. The information given in Table 15 gives descriptive statistical data, including mean, standard deviation and $95 \%$ confidence intervals for the dependent variable- click through the score for each of the categories of the advertisement on Google where all the 12 possibilities (weekday/weekend) with timings have been listed.

Table 12 is the output of the ANOVA analysis which is used to test the Hypothesis (C) to understand if there is a statistically significant difference between the group means of click through ratio and the day and time during which the advertisement was displayed on Google. It can be inferred from the significance value 0.000 (i.e., $p=0.000$ ), which is below 0.05 , that there is a statistically significant difference between click through ratio and day and time of the advertisement. This indicates that different advertisement displayed on Google at different points of time have different click through ratios. To know which of those specific advertisement differed in the click through ratio, Tukey post hoc test was used and Table 17 gives the output including the multiple comparisons table for the format type. From Table 17, it can be inferred that there is a statistically significant difference in the click through ratio of the advertisement that were added during the Week Day 00.00 am to 08.00 am in comparison with Advertisement displayed during any other day or time $(\mathrm{p}=0.000)$. There is a statistically significant difference in click through ratio of the advertisement that were added during all the 12 categories of day or time in comparison with advertisement displayed during any other day or time $(\mathrm{p}=0.000)$. The Table 18 gives the details of the day and time during which there is no statistically significant difference in the click through ratio of the advertisement when compared with other days and timings. 
Table 13. ANOVA analysis - DAY_TIME

Time click through ratio

\begin{tabular}{cccccc}
\hline & Sum of Squares & df & $\begin{array}{c}\text { Mean } \\
\text { Square }\end{array}$ & F & Sig. \\
\hline Between Groups & 30.894 & 11 & 2.809 & 37607.858 & .000 \\
Within Groups & .089 & 1188 & .000 & & \\
Total & 30.983 & 1199 & & \\
\hline
\end{tabular}

Table 14. Multiple comparisons of DayTime

\section{Dependent Variable: Time click through ratio \\ Tukey HSD}

$\begin{array}{lllll}\text { (I) Time } & \text { (J) Time } & \text { Mean } & \text { Std. Error } & \text { Sig. }\end{array}$

\begin{tabular}{|c|c|c|c|c|c|c|}
\hline & & $\begin{array}{l}\text { Difference } \\
\quad(\mathrm{I}-\mathrm{J})\end{array}$ & & & $\begin{array}{l}\text { Lower } \\
\text { Bound }\end{array}$ & Upper Bound \\
\hline \multirow[t]{11}{*}{$\begin{array}{l}\text { Week Day } 00.00 \\
\text { am to } 08.00 \mathrm{am}\end{array}$} & $\begin{array}{l}\text { Week Day } 08.01 \\
\text { am to } 10.00 \mathrm{am}\end{array}$ & $-.1429000^{*}$ & .0012221 & .000 & -.146902 & -.138898 \\
\hline & $\begin{array}{l}\text { Week Day } 10.01 \\
\text { am to } 05 \# 00 \mathrm{pm}\end{array}$ & $-.5034910^{*}$ & .0012221 & .000 & -.507493 & -.499489 \\
\hline & $\begin{array}{l}\text { Week Day } 05.01 \\
\text { pm to } 08.00 \mathrm{pm}\end{array}$ & $-.1606250^{*}$ & .0012221 & .000 & -.164627 & -.156623 \\
\hline & $\begin{array}{l}\text { Week Day } 08.01 \\
\text { pm to } 10.00 \mathrm{pm}\end{array}$ & $-.0480940^{*}$ & .0012221 & .000 & -.052096 & -.044092 \\
\hline & $\begin{array}{l}\text { Week Day } 10.00 \\
\text { pm to } 11.59 \mathrm{pm}\end{array}$ & $.0130740^{*}$ & .0012221 & .000 & .009072 & .017076 \\
\hline & $\begin{array}{l}\text { Week End } 00.00 \\
\text { am to } 08.00 \mathrm{am}\end{array}$ & $.0967960^{*}$ & .0012221 & .000 & .092794 & .100798 \\
\hline & $\begin{array}{l}\text { Week End } 08.01 \\
\text { am to } 10.00 \mathrm{am}\end{array}$ & $-.1195800^{*}$ & .0012221 & .000 & -.123582 & -.115578 \\
\hline & $\begin{array}{l}\text { Week End } 10.01 \\
\text { am to } 05 \# 00 \mathrm{pm}\end{array}$ & $-.2506870^{*}$ & .0012221 & .000 & -.254689 & -.246685 \\
\hline & $\begin{array}{l}\text { Week End } 05.01 \\
\mathrm{pm} \text { to } 08.00 \mathrm{pm}\end{array}$ & $-.0951360^{*}$ & .0012221 & .000 & -.099138 & -.091134 \\
\hline & $\begin{array}{l}\text { Week End } 08.01 \\
\text { pm to } 10.00 \mathrm{pm}\end{array}$ & $.0444060^{*}$ & .0012221 & .000 & .040404 & .048408 \\
\hline & $\begin{array}{l}\text { Week End } 10.00 \\
\text { pm to } 11.59 \mathrm{pm}\end{array}$ & $.0832620^{*}$ & .0012221 & .000 & .079260 & .087264 \\
\hline \multirow[t]{5}{*}{$\begin{array}{l}\text { Week Day } 08.01 \\
\text { am to } 10.00 \mathrm{am}\end{array}$} & $\begin{array}{l}\text { Week Day } 00.00 \\
\text { am to } 08.00 \mathrm{am}\end{array}$ & $.1429000^{*}$ & .0012221 & .000 & .138898 & .146902 \\
\hline & $\begin{array}{l}\text { Week Day } 10.01 \\
\text { am to } 05 \# 00 \mathrm{pm}\end{array}$ & $-.3605910^{*}$ & .0012221 & .000 & -.364593 & -.356589 \\
\hline & $\begin{array}{l}\text { Week Day } 05.01 \\
\text { pm to } 08.00 \mathrm{pm}\end{array}$ & $-.0177250^{*}$ & .0012221 & .000 & -.021727 & -.013723 \\
\hline & $\begin{array}{l}\text { Week Day } 08.01 \\
\text { pm to } 10.00 \mathrm{pm}\end{array}$ & $.0948060^{*}$ & .0012221 & .000 & .090804 & .098808 \\
\hline & $\begin{array}{l}\text { Week Day } 10.00 \\
\text { pm to } 11.59 \mathrm{pm}\end{array}$ & $.1559740^{*}$ & .0012221 & .000 & .151972 & .159976 \\
\hline
\end{tabular}




\begin{tabular}{|c|c|c|c|c|c|c|}
\hline & $\begin{array}{l}\text { Week End } 00.00 \\
\text { am to } 08.00 \mathrm{am}\end{array}$ & $.2396960^{*}$ & .0012221 & .000 & .235694 & .243698 \\
\hline & $\begin{array}{l}\text { Week End } 08.01 \\
\text { am to } 10.00 \mathrm{am}\end{array}$ & $.0233200^{*}$ & .0012221 & .000 & .019318 & .027322 \\
\hline & $\begin{array}{l}\text { Week End } 10.01 \\
\text { am to } 05 \# 00 \mathrm{pm}\end{array}$ & $-.1077870^{*}$ & .0012221 & .000 & -.111789 & -.103785 \\
\hline & $\begin{array}{l}\text { Week End } 05.01 \\
\text { pm to } 08.00 \mathrm{pm}\end{array}$ & $.0477640^{*}$ & .0012221 & .000 & .043762 & .051766 \\
\hline & $\begin{array}{l}\text { Week End } 08.01 \\
\text { pm to } 10.00 \mathrm{pm}\end{array}$ & $.1873060^{*}$ & .0012221 & .000 & .183304 & .191308 \\
\hline & $\begin{array}{l}\text { Week End } 10.00 \\
\text { pm to } 11.59 \mathrm{pm}\end{array}$ & $.2261620^{*}$ & .0012221 & .000 & .222160 & .230164 \\
\hline \multirow[t]{11}{*}{$\begin{array}{l}\text { Week Day } 10.01 \\
\text { am to } 05 \# 00 \mathrm{pm}\end{array}$} & $\begin{array}{l}\text { Week Day } 00.00 \\
\text { am to } 08.00 \mathrm{am}\end{array}$ & $.5034910^{*}$ & .0012221 & .000 & .499489 & .507493 \\
\hline & $\begin{array}{l}\text { Week Day } 08.01 \\
\text { am to } 10.00 \mathrm{am}\end{array}$ & $.3605910^{*}$ & .0012221 & .000 & .356589 & .364593 \\
\hline & $\begin{array}{l}\text { Week Day } 05.01 \\
\text { pm to } 08.00 \mathrm{pm}\end{array}$ & $.3428660^{*}$ & .0012221 & .000 & .338864 & .346868 \\
\hline & $\begin{array}{l}\text { Week Day } 08.01 \\
\text { pm to } 10.00 \mathrm{pm}\end{array}$ & $.4553970^{*}$ & .0012221 & .000 & .451395 & .459399 \\
\hline & $\begin{array}{l}\text { Week Day } 10.00 \\
\text { pm to } 11.59 \mathrm{pm}\end{array}$ & $.5165650^{*}$ & .0012221 & .000 & .512563 & .520567 \\
\hline & $\begin{array}{l}\text { Week End } 00.00 \\
\text { am to } 08.00 \mathrm{am}\end{array}$ & $.6002870^{*}$ & .0012221 & .000 & .596285 & .604289 \\
\hline & $\begin{array}{l}\text { Week End } 08.01 \\
\text { am to } 10.00 \mathrm{am}\end{array}$ & $.3839110^{*}$ & .0012221 & .000 & .379909 & .387913 \\
\hline & $\begin{array}{l}\text { Week End } 10.01 \\
\text { am to } 05 \# 00 \mathrm{pm}\end{array}$ & $.2528040^{*}$ & .0012221 & .000 & .248802 & .256806 \\
\hline & $\begin{array}{l}\text { Week End } 05.01 \\
\text { pm to } 08.00 \mathrm{pm}\end{array}$ & $.4083550^{*}$ & .0012221 & .000 & .404353 & .412357 \\
\hline & $\begin{array}{l}\text { Week End } 08.01 \\
\text { pm to } 10.00 \mathrm{pm}\end{array}$ & $.5478970^{*}$ & .0012221 & .000 & .543895 & .551899 \\
\hline & $\begin{array}{l}\text { Week End } 10.00 \\
\text { pm to } 11.59 \mathrm{pm}\end{array}$ & $.5867530^{*}$ & .0012221 & .000 & .582751 & .590755 \\
\hline \multirow[t]{8}{*}{$\begin{array}{l}\text { Week Day } 05.01 \\
\text { pm to } 08.00 \mathrm{pm}\end{array}$} & $\begin{array}{l}\text { Week Day } 00.00 \\
\text { am to } 08.00 \mathrm{am}\end{array}$ & $.1606250^{*}$ & .0012221 & .000 & .156623 & .164627 \\
\hline & $\begin{array}{l}\text { Week Day } 08.01 \\
\text { am to } 10.00 \mathrm{am}\end{array}$ & $.0177250^{*}$ & .0012221 & .000 & .013723 & .021727 \\
\hline & $\begin{array}{l}\text { Week Day } 10.01 \\
\text { am to } 05 \# 00 \mathrm{pm}\end{array}$ & $-.3428660^{*}$ & .0012221 & .000 & -.346868 & -.338864 \\
\hline & $\begin{array}{l}\text { Week Day } 08.01 \\
\text { pm to } 10.00 \mathrm{pm}\end{array}$ & $.1125310^{*}$ & .0012221 & .000 & .108529 & .116533 \\
\hline & $\begin{array}{l}\text { Week Day } 10.00 \\
\text { pm to } 11.59 \mathrm{pm}\end{array}$ & $.1736990^{*}$ & .0012221 & .000 & .169697 & .177701 \\
\hline & $\begin{array}{l}\text { Week End } 00.00 \\
\text { am to } 08.00 \mathrm{am}\end{array}$ & $.2574210^{*}$ & .0012221 & .000 & .253419 & .261423 \\
\hline & $\begin{array}{l}\text { Week End } 08.01 \\
\text { am to } 10.00 \mathrm{am}\end{array}$ & $.0410450^{*}$ & .0012221 & .000 & .037043 & .045047 \\
\hline & $\begin{array}{l}\text { Week End } 10.01 \\
\text { am to } 05 \# 00 \mathrm{pm}\end{array}$ & $-.0900620^{*}$ & .0012221 & .000 & -.094064 & -.086060 \\
\hline
\end{tabular}




\begin{tabular}{|c|c|c|c|c|c|c|}
\hline & $\begin{array}{l}\text { Week End } 05.01 \\
\text { pm to } 08.00 \mathrm{pm}\end{array}$ & $.0654890^{*}$ & .0012221 & .000 & .061487 & .069491 \\
\hline & $\begin{array}{l}\text { Week End } 08.01 \\
\text { pm to } 10.00 \mathrm{pm}\end{array}$ & $.2050310^{*}$ & .0012221 & .000 & .201029 & .209033 \\
\hline & $\begin{array}{l}\text { Week End } 10.00 \\
\text { pm to } 11.59 \mathrm{pm}\end{array}$ & $.2438870^{*}$ & .0012221 & .000 & .239885 & .247889 \\
\hline \multirow[t]{11}{*}{$\begin{array}{l}\text { Week Day } 08.01 \\
\text { pm to } 10.00 \mathrm{pm}\end{array}$} & $\begin{array}{l}\text { Week Day } 00.00 \\
\text { am to } 08.00 \mathrm{am}\end{array}$ & $.0480940^{*}$ & .0012221 & .000 & .044092 & .052096 \\
\hline & $\begin{array}{l}\text { Week Day } 08.01 \\
\text { am to } 10.00 \mathrm{am}\end{array}$ & $-.0948060^{*}$ & .0012221 & .000 & -.098808 & -.090804 \\
\hline & $\begin{array}{l}\text { Week Day } 10.01 \\
\text { am to } 05 \# 00 \mathrm{pm}\end{array}$ & $-.4553970^{*}$ & .0012221 & .000 & -.459399 & -.451395 \\
\hline & $\begin{array}{l}\text { Week Day } 05.01 \\
\text { pm to } 08.00 \mathrm{pm}\end{array}$ & $-.1125310^{*}$ & .0012221 & .000 & -.116533 & -.108529 \\
\hline & $\begin{array}{l}\text { Week Day } 10.00 \\
\text { pm to } 11.59 \mathrm{pm}\end{array}$ & $.0611680^{*}$ & .0012221 & .000 & .057166 & .065170 \\
\hline & $\begin{array}{l}\text { Week End } 00.00 \\
\text { am to } 08.00 \mathrm{am}\end{array}$ & $.1448900^{*}$ & .0012221 & .000 & .140888 & .148892 \\
\hline & $\begin{array}{l}\text { Week End } 08.01 \\
\text { am to } 10.00 \mathrm{am}\end{array}$ & $-.0714860^{*}$ & .0012221 & .000 & -.075488 & -.067484 \\
\hline & $\begin{array}{l}\text { Week End } 10.01 \\
\text { am to } 05 \# 00 \mathrm{pm}\end{array}$ & $-.2025930^{*}$ & .0012221 & .000 & -.206595 & -.198591 \\
\hline & $\begin{array}{l}\text { Week End } 05.01 \\
\text { pm to } 08.00 \mathrm{pm}\end{array}$ & $-.0470420^{*}$ & .0012221 & .000 & -.051044 & -.043040 \\
\hline & $\begin{array}{l}\text { Week End } 08.01 \\
\text { pm to } 10.00 \mathrm{pm}\end{array}$ & $.0925000^{*}$ & .0012221 & .000 & .088498 & .096502 \\
\hline & $\begin{array}{l}\text { Week End } 10.00 \\
\text { pm to } 11.59 \mathrm{pm}\end{array}$ & $.1313560^{*}$ & .0012221 & .000 & .127354 & .135358 \\
\hline \multirow[t]{11}{*}{$\begin{array}{l}\text { Week Day } 10.00 \\
\text { pm to } 11.59 \mathrm{pm}\end{array}$} & $\begin{array}{l}\text { Week Day } 00.00 \\
\text { am to } 08.00 \mathrm{am}\end{array}$ & $-.0130740^{*}$ & .0012221 & .000 & -.017076 & -.009072 \\
\hline & $\begin{array}{l}\text { Week Day } 08.01 \\
\text { am to } 10.00 \mathrm{am}\end{array}$ & $-.1559740^{*}$ & .0012221 & .000 & -.159976 & -.151972 \\
\hline & $\begin{array}{l}\text { Week Day } 10.01 \\
\text { am to } 05 \# 00 \mathrm{pm}\end{array}$ & $-.5165650^{*}$ & .0012221 & .000 & -.520567 & -.512563 \\
\hline & $\begin{array}{l}\text { Week Day } 05.01 \\
\text { pm to } 08.00 \mathrm{pm}\end{array}$ & $-.1736990^{*}$ & .0012221 & .000 & -.177701 & -.169697 \\
\hline & $\begin{array}{l}\text { Week Day } 08.01 \\
\text { pm to } 10.00 \mathrm{pm}\end{array}$ & $-.0611680^{*}$ & .0012221 & .000 & -.065170 & -.057166 \\
\hline & $\begin{array}{l}\text { Week End } 00.00 \\
\text { am to } 08.00 \mathrm{am}\end{array}$ & $.0837220^{*}$ & .0012221 & .000 & .079720 & .087724 \\
\hline & $\begin{array}{l}\text { Week End } 08.01 \\
\text { am to } 10.00 \mathrm{am}\end{array}$ & $-.1326540^{*}$ & .0012221 & .000 & -.136656 & -.128652 \\
\hline & $\begin{array}{l}\text { Week End } 10.01 \\
\text { am to } 05 \# 00 \mathrm{pm}\end{array}$ & $-.2637610^{*}$ & .0012221 & .000 & -.267763 & -.259759 \\
\hline & $\begin{array}{l}\text { Week End } 05.01 \\
\text { pm to } 08.00 \mathrm{pm}\end{array}$ & $-.1082100^{*}$ & .0012221 & .000 & -.112212 & -.104208 \\
\hline & $\begin{array}{l}\text { Week End } 08.01 \\
\text { pm to } 10.00 \mathrm{pm}\end{array}$ & $.0313320^{*}$ & .0012221 & .000 & .027330 & .035334 \\
\hline & $\begin{array}{l}\text { Week End } 10.00 \\
\text { pm to } 11.59 \mathrm{pm}\end{array}$ & $.0701880^{*}$ & .0012221 & .000 & .066186 & .074190 \\
\hline
\end{tabular}




\begin{tabular}{|c|c|c|c|c|c|c|}
\hline \multirow[t]{11}{*}{$\begin{array}{l}\text { Week End 00.00 } \\
\text { am to } 08.00 \mathrm{am}\end{array}$} & $\begin{array}{l}\text { Week Day } 00.00 \\
\text { am to } 08.00 \mathrm{am}\end{array}$ & $-.0967960^{*}$ & .0012221 & .000 & -.100798 & -.092794 \\
\hline & $\begin{array}{l}\text { Week Day } 08.01 \\
\text { am to } 10.00 \mathrm{am}\end{array}$ & $-.2396960^{*}$ & .0012221 & .000 & -.243698 & -.235694 \\
\hline & $\begin{array}{l}\text { Week Day } 10.01 \\
\text { am to } 05 \# 00 \mathrm{pm}\end{array}$ & $-.6002870^{*}$ & .0012221 & .000 & -.604289 & -.596285 \\
\hline & $\begin{array}{l}\text { Week Day } 05.01 \\
\text { pm to } 08.00 \mathrm{pm}\end{array}$ & $-.2574210^{*}$ & .0012221 & .000 & -.261423 & -.253419 \\
\hline & $\begin{array}{l}\text { Week Day } 08.01 \\
\text { pm to } 10.00 \mathrm{pm}\end{array}$ & $-.1448900^{*}$ & .0012221 & .000 & -.148892 & -.140888 \\
\hline & $\begin{array}{l}\text { Week Day } 10.00 \\
\text { pm to } 11.59 \mathrm{pm}\end{array}$ & $-.0837220^{*}$ & .0012221 & .000 & -.087724 & -.079720 \\
\hline & $\begin{array}{l}\text { Week End } 08.01 \\
\text { am to } 10.00 \mathrm{am}\end{array}$ & $-.2163760^{*}$ & .0012221 & .000 & -.220378 & -.212374 \\
\hline & $\begin{array}{l}\text { Week End } 10.01 \\
\text { am to } 05 \# 00 \mathrm{pm}\end{array}$ & $-.3474830^{*}$ & .0012221 & .000 & -.351485 & -.343481 \\
\hline & $\begin{array}{l}\text { Week End } 05.01 \\
\text { pm to } 08.00 \mathrm{pm}\end{array}$ & $-.1919320^{*}$ & .0012221 & .000 & -.195934 & -.187930 \\
\hline & $\begin{array}{l}\text { Week End } 08.01 \\
\text { pm to } 10.00 \mathrm{pm}\end{array}$ & $-.0523900^{*}$ & .0012221 & .000 & -.056392 & -.048388 \\
\hline & $\begin{array}{l}\text { Week End } 10.00 \\
\text { pm to } 11.59 \mathrm{pm}\end{array}$ & $-.0135340^{*}$ & .0012221 & .000 & -.017536 & -.009532 \\
\hline \multirow[t]{11}{*}{$\begin{array}{l}\text { Week End } 08.01 \\
\text { am to } 10.00 \text { am }\end{array}$} & $\begin{array}{l}\text { Week Day } 00.00 \\
\text { am to } 08.00 \mathrm{am}\end{array}$ & $.1195800^{*}$ & .0012221 & .000 & .115578 & .123582 \\
\hline & $\begin{array}{l}\text { Week Day } 08.01 \\
\text { am to } 10.00 \mathrm{am}\end{array}$ & $-.0233200^{*}$ & .0012221 & .000 & -.027322 & -.019318 \\
\hline & $\begin{array}{l}\text { Week Day } 10.01 \\
\text { am to } 05 \# 00 \mathrm{pm}\end{array}$ & $-.3839110^{*}$ & .0012221 & .000 & -.387913 & -.379909 \\
\hline & $\begin{array}{l}\text { Week Day } 05.01 \\
\text { pm to } 08.00 \mathrm{pm}\end{array}$ & $-.0410450^{*}$ & .0012221 & .000 & -.045047 & -.037043 \\
\hline & $\begin{array}{l}\text { Week Day } 08.01 \\
\text { pm to } 10.00 \mathrm{pm}\end{array}$ & $.0714860^{*}$ & .0012221 & .000 & .067484 & .075488 \\
\hline & $\begin{array}{l}\text { Week Day } 10.00 \\
\text { pm to } 11.59 \mathrm{pm}\end{array}$ & $.1326540^{*}$ & .0012221 & .000 & .128652 & .136656 \\
\hline & $\begin{array}{l}\text { Week End } 00.00 \\
\text { am to } 08.00 \mathrm{am}\end{array}$ & $.2163760^{*}$ & .0012221 & .000 & .212374 & .220378 \\
\hline & $\begin{array}{l}\text { Week End } 10.01 \\
\text { am to } 05 \# 00 \mathrm{pm}\end{array}$ & $-.1311070^{*}$ & .0012221 & .000 & -.135109 & -.127105 \\
\hline & $\begin{array}{l}\text { Week End } 05.01 \\
\text { pm to } 08.00 \mathrm{pm}\end{array}$ & $.0244440^{*}$ & .0012221 & .000 & .020442 & .028446 \\
\hline & $\begin{array}{l}\text { Week End } 08.01 \\
\mathrm{pm} \text { to } 10.00 \mathrm{pm}\end{array}$ & $.1639860^{*}$ & .0012221 & .000 & .159984 & .167988 \\
\hline & $\begin{array}{l}\text { Week End } 10.00 \\
\text { pm to } 11.59 \mathrm{pm}\end{array}$ & $.2028420^{*}$ & .0012221 & .000 & .198840 & .206844 \\
\hline \multirow[t]{3}{*}{$\begin{array}{l}\text { Week End } 10.01 \\
\text { am to } 05 \# 00 \mathrm{pm}\end{array}$} & $\begin{array}{l}\text { Week Day } 00.00 \\
\text { am to } 08.00 \mathrm{am}\end{array}$ & $.2506870^{*}$ & .0012221 & .000 & .246685 & .254689 \\
\hline & $\begin{array}{l}\text { Week Day } 08.01 \\
\text { am to } 10.00 \mathrm{am}\end{array}$ & $.1077870^{*}$ & .0012221 & .000 & .103785 & .111789 \\
\hline & $\begin{array}{l}\text { Week Day } 10.01 \\
\text { am to } 05 \# 00 \mathrm{pm}\end{array}$ & $-.2528040^{*}$ & .0012221 & .000 & -.256806 & -.248802 \\
\hline
\end{tabular}




\begin{tabular}{|c|c|c|c|c|c|c|}
\hline & $\begin{array}{l}\text { Week Day } 05.01 \\
\text { pm to } 08.00 \mathrm{pm}\end{array}$ & $.0900620^{*}$ & .0012221 & .000 & .086060 & .094064 \\
\hline & $\begin{array}{l}\text { Week Day } 08.01 \\
\text { pm to } 10.00 \mathrm{pm}\end{array}$ & $.2025930^{*}$ & .0012221 & .000 & .198591 & .206595 \\
\hline & $\begin{array}{l}\text { Week Day } 10.00 \\
\text { pm to } 11.59 \mathrm{pm}\end{array}$ & $.2637610^{*}$ & .0012221 & .000 & .259759 & .267763 \\
\hline & $\begin{array}{l}\text { Week End } 00.00 \\
\text { am to } 08.00 \mathrm{am}\end{array}$ & $.3474830^{*}$ & .0012221 & .000 & .343481 & .351485 \\
\hline & $\begin{array}{l}\text { Week End } 08.01 \\
\text { am to } 10.00 \mathrm{am}\end{array}$ & $.1311070^{*}$ & .0012221 & .000 & .127105 & .135109 \\
\hline & $\begin{array}{l}\text { Week End } 05.01 \\
\text { pm to } 08.00 \mathrm{pm}\end{array}$ & $.1555510^{*}$ & .0012221 & .000 & .151549 & .159553 \\
\hline & $\begin{array}{l}\text { Week End } 08.01 \\
\text { pm to } 10.00 \mathrm{pm}\end{array}$ & $.2950930^{*}$ & .0012221 & .000 & .291091 & .299095 \\
\hline & $\begin{array}{l}\text { Week End } 10.00 \\
\text { pm to } 11.59 \mathrm{pm}\end{array}$ & $.3339490^{*}$ & .0012221 & .000 & .329947 & .337951 \\
\hline $\begin{array}{l}\text { Week End } 05.01 \\
\text { pm to } 08.00 \mathrm{pm}\end{array}$ & $\begin{array}{l}\text { Week Day } 00.00 \\
\text { am to } 08.00 \mathrm{am}\end{array}$ & $.0951360^{*}$ & .0012221 & .000 & .091134 & .099138 \\
\hline & $\begin{array}{l}\text { Week Day } 08.01 \\
\text { am to } 10.00 \mathrm{am}\end{array}$ & $-.0477640^{*}$ & .0012221 & .000 & -.051766 & -.043762 \\
\hline & $\begin{array}{l}\text { Week Day } 10.01 \\
\text { am to } 05 \# 00 \mathrm{pm}\end{array}$ & $-.4083550^{*}$ & .0012221 & .000 & -.412357 & -.404353 \\
\hline & $\begin{array}{l}\text { Week Day } 05.01 \\
\text { pm to } 08.00 \mathrm{pm}\end{array}$ & $-.0654890^{*}$ & .0012221 & .000 & -.069491 & -.061487 \\
\hline & $\begin{array}{l}\text { Week Day } 08.01 \\
\text { pm to } 10.00 \mathrm{pm}\end{array}$ & $.0470420^{*}$ & .0012221 & .000 & .043040 & .051044 \\
\hline & $\begin{array}{l}\text { Week Day } 10.00 \\
\text { pm to } 11.59 \mathrm{pm}\end{array}$ & $.1082100^{*}$ & .0012221 & .000 & .104208 & .112212 \\
\hline & $\begin{array}{l}\text { Week End } 00.00 \\
\text { am to } 08.00 \mathrm{am}\end{array}$ & $.1919320^{*}$ & .0012221 & .000 & .187930 & .195934 \\
\hline & $\begin{array}{l}\text { Week End } 08.01 \\
\text { am to } 10.00 \mathrm{am}\end{array}$ & $-.0244440^{*}$ & .0012221 & .000 & -.028446 & -.020442 \\
\hline & $\begin{array}{l}\text { Week End } 10.01 \\
\text { am to } 05 \# 00 \mathrm{pm}\end{array}$ & $-.1555510^{*}$ & .0012221 & .000 & -.159553 & -.151549 \\
\hline & $\begin{array}{l}\text { Week End } 08.01 \\
\text { pm to } 10.00 \mathrm{pm}\end{array}$ & $.1395420^{*}$ & .0012221 & .000 & .135540 & .143544 \\
\hline & $\begin{array}{l}\text { Week End } 10.00 \\
\text { pm to } 11.59 \mathrm{pm}\end{array}$ & $.1783980^{*}$ & .0012221 & .000 & .174396 & .182400 \\
\hline $\begin{array}{l}\text { Week End } 08.01 \\
\text { pm to } 10.00 \mathrm{pm}\end{array}$ & $\begin{array}{l}\text { Week Day } 00.00 \\
\text { am to } 08.00 \mathrm{am}\end{array}$ & $-.0444060^{*}$ & .0012221 & .000 & -.048408 & -.040404 \\
\hline & $\begin{array}{l}\text { Week Day } 08.01 \\
\text { am to } 10.00 \mathrm{am}\end{array}$ & $-.1873060^{*}$ & .0012221 & .000 & -.191308 & -.183304 \\
\hline & $\begin{array}{l}\text { Week Day } 10.01 \\
\text { am to } 05 \# 00 \mathrm{pm}\end{array}$ & $-.5478970^{*}$ & .0012221 & .000 & -.551899 & -.543895 \\
\hline & $\begin{array}{l}\text { Week Day } 05.01 \\
\text { pm to } 08.00 \mathrm{pm}\end{array}$ & $-.2050310^{*}$ & .0012221 & .000 & -.209033 & -.201029 \\
\hline & $\begin{array}{l}\text { Week Day } 08.01 \\
\text { pm to } 10.00 \mathrm{pm}\end{array}$ & $-.0925000^{*}$ & .0012221 & .000 & -.096502 & -.088498 \\
\hline & $\begin{array}{l}\text { Week Day } 10.00 \\
\text { pm to } 11.59 \mathrm{pm}\end{array}$ & $-.0313320^{*}$ & .0012221 & .000 & -.035334 & -.027330 \\
\hline
\end{tabular}




\begin{tabular}{|c|c|c|c|c|c|c|}
\hline & $\begin{array}{l}\text { Week End } 00.00 \\
\text { am to } 08.00 \mathrm{am}\end{array}$ & $.0523900^{*}$ & .0012221 & .000 & .048388 & .056392 \\
\hline & $\begin{array}{l}\text { Week End } 08.01 \\
\text { am to } 10.00 \mathrm{am}\end{array}$ & $-.1639860^{*}$ & .0012221 & .000 & -.167988 & -.159984 \\
\hline & $\begin{array}{l}\text { Week End } 10.01 \\
\text { am to } 05 \# 00 \mathrm{pm}\end{array}$ & $-.2950930^{*}$ & .0012221 & .000 & -.299095 & -.291091 \\
\hline & $\begin{array}{l}\text { Week End } 05.01 \\
\text { pm to } 08.00 \mathrm{pm}\end{array}$ & $-.1395420^{*}$ & .0012221 & .000 & -.143544 & -.135540 \\
\hline & $\begin{array}{l}\text { Week End } 10.00 \\
\text { pm to } 11.59 \mathrm{pm}\end{array}$ & $.0388560^{*}$ & .0012221 & .000 & .034854 & .042858 \\
\hline \multirow[t]{11}{*}{$\begin{array}{l}\text { Week End } 10.00 \\
\text { pm to } 11.59 \text { pm }\end{array}$} & $\begin{array}{l}\text { Week Day } 00.00 \\
\text { am to } 08.00 \mathrm{am}\end{array}$ & $-.0832620^{*}$ & .0012221 & .000 & -.087264 & -.079260 \\
\hline & $\begin{array}{l}\text { Week Day } 08.01 \\
\text { am to } 10.00 \mathrm{am}\end{array}$ & $-.2261620^{*}$ & .0012221 & .000 & -.230164 & -.222160 \\
\hline & $\begin{array}{l}\text { Week Day } 10.01 \\
\text { am to } 05 \# 00 \mathrm{pm}\end{array}$ & $-.5867530^{*}$ & .0012221 & .000 & -.590755 & -.582751 \\
\hline & $\begin{array}{l}\text { Week Day } 05.01 \\
\text { pm to } 08.00 \mathrm{pm}\end{array}$ & $-.2438870^{*}$ & .0012221 & .000 & -.247889 & -.239885 \\
\hline & $\begin{array}{l}\text { Week Day } 08.01 \\
\text { pm to } 10.00 \mathrm{pm}\end{array}$ & $-.1313560^{*}$ & .0012221 & .000 & -.135358 & -.127354 \\
\hline & $\begin{array}{l}\text { Week Day } 10.00 \\
\text { pm to } 11.59 \mathrm{pm}\end{array}$ & $-.0701880^{*}$ & .0012221 & .000 & -.074190 & -.066186 \\
\hline & $\begin{array}{l}\text { Week End } 00.00 \\
\text { am to } 08.00 \mathrm{am}\end{array}$ & $.0135340^{*}$ & .0012221 & .000 & .009532 & .017536 \\
\hline & $\begin{array}{l}\text { Week End } 08.01 \\
\text { am to } 10.00 \mathrm{am}\end{array}$ & $-.2028420^{*}$ & .0012221 & .000 & -.206844 & -.198840 \\
\hline & $\begin{array}{l}\text { Week End } 10.01 \\
\text { am to } 05 \# 00 \mathrm{pm}\end{array}$ & $-.3339490^{*}$ & .0012221 & .000 & -.337951 & -.329947 \\
\hline & $\begin{array}{l}\text { Week End } 05.01 \\
\text { pm to } 08.00 \mathrm{pm}\end{array}$ & $-.1783980^{*}$ & .0012221 & .000 & -.182400 & -.174396 \\
\hline & $\begin{array}{l}\text { Week End } 08.01 \\
\text { pm to } 10.00 \mathrm{pm}\end{array}$ & $-.0388560^{*}$ & .0012221 & .000 & -.042858 & -.034854 \\
\hline
\end{tabular}

*. The mean difference is significant at the 0.05 level.

Table 15. No significant difference in click through ratio for advertisement - DAY_ TIME

Format type
Statistically NO significant difference in click through ratio in advertisements posted during the following day and time

\begin{tabular}{ll}
\hline Week Day 00.00 am to 08.00 am & NIL \\
Week Day 08.01 am to 10.00 am & NIL \\
Week Day 10.01 am to $05 \# 00$ pm & NIL \\
Week Day 05.01 pm to 08.00 pm & NIL \\
Week Day 08.01 pm to 10.00 pm & NIL \\
Week Day 10.00 pm to 11.59 pm & NIL \\
Week End 00.00 am to 08.00 am & NIL \\
Week End 08.01 am to 10.00 am & NIL \\
Week End 10.01 am to $05 \# 00$ pm & NIL \\
\hline
\end{tabular}




$\begin{array}{ll}\text { Week End } 05.01 \mathrm{pm} \text { to } 08.00 \mathrm{pm} & \mathrm{NIL} \\ \text { Week End } 08.01 \mathrm{pm} \text { to } 10.00 \mathrm{pm} & \mathrm{NIL} \\ \text { Week End } 10.00 \mathrm{pm} \text { to } 11.59 \mathrm{pm} & \mathrm{NIL}\end{array}$

Table 16. Intensity index of Day_Time- click through ratio

\begin{tabular}{llll}
\hline & N & Mean & Std. Deviation \\
\hline Week Day 10.01 am to 05\#00 pm & 100 & .653162 & .0120479 \\
Week End 10.01 am to 05\#00 pm & 100 & .400358 & .0074065 \\
Week Day 05.01 pm to 08.00 pm & 100 & .310296 & .0014062 \\
Week Day 08.01 am to 10.00 am & 100 & .292571 & .0043112 \\
Week End 08.01 am to 10.00 am & 100 & .269251 & .0115473 \\
Week End 05.01 pm to 08.00 pm & 100 & .244807 & .0110917 \\
Week Day 08.01 pm to 10.00 pm & 100 & .197765 & .0109310 \\
Week Day 00.00 am to 08.00 am & 100 & .149671 & .0093841 \\
Week Day 10.00 pm to 11.59 pm & 100 & .136597 & .0132028 \\
Week End 08.01 pm to 10.00 pm & 100 & .105265 & .0037481 \\
Week End 10.00 pm to 11.59 pm & 100 & .066409 & .0045813 \\
Week End 00.00 am to 08.00 am & 100 & .052875 & .0015227 \\
\hline
\end{tabular}

Table No 16 Indicates intensity index of the Days and Time of advertisement. It shows that Weekdays 10.01 am to $05.00 \mathrm{pm}(\mathrm{M}=.653162)$, Weekends $10.01 \mathrm{am}$ to $05.00 \mathrm{pm}(\mathrm{M}=.400358)$ and Weekdays $05.01 \mathrm{pm}$ to $08.00 \mathrm{pm}$ $(\mathrm{M}=.310296)$ advertisement have the highest click though ratio while Weekends $08.01 \mathrm{pm}$ to $10.00 \mathrm{pm}$ $(\mathrm{M}=.105265)$, Weekends $10.00 \mathrm{pm}$ to $11.59 \mathrm{pm}(\mathrm{M}=.066409)$ and Weekends $00.00 \mathrm{am}$ to $08.00 \mathrm{am}(\mathrm{M}=.052875)$ have lower click though ratio. It can be concluded that Weekdays $10.01 \mathrm{am}$ to $05.00 \mathrm{pm}$, Weekends $10.01 \mathrm{am}$ to $05.00 \mathrm{pm}$ and Weekdays $05.01 \mathrm{pm}$ to $08.00 \mathrm{pm}$ have a higher click through ratio while Weekends $08.01 \mathrm{pm}$ to $10.00 \mathrm{pm}$, Weekends $10.00 \mathrm{pm}$ to $11.59 \mathrm{pm}$ and Weekends $00.00 \mathrm{am}$ to 08.00 am have lower click though ratio.

\section{Conclusion and Discussions}

From the study conducted, the importance, impact and influence of digital advertisement for B schools can be clearly understood. It has also been found that different tools generate different results for the marketing process. Based on the research findings, the following strategies can be employed to provide a framework while designing the digital marketing campaigns:

Strategy 1: Selectively create run specific advertisement on Google for better impressions and clicks

The results suggest that different categories of advertisement have different click through ratios and that advertisement belonging to the admissions category had a higher CTR as compared to all other categories (Table 7 denotes the statistical significance). Hence, for a higher CTR, having admission-specific advertisement can prove beneficial.

Strategy 2: A proper format of advertisement should be chosen or created to have a good CTR.

The ANOVA results indicate that the CTR generated by advertisement differed based on their format (statistical significance shared above). The advertisement formats that have a higher CTR include: extensions, dynamic text, text, interactive video, text $\&$ phone number and short videos (reflected in Table 11 and 12).

Strategy 3: In order to ensure high CTR and reach, an effective time and day to run the advertisement should be chosen.

The day and timing of an advertisement plays an important factor in the CTR that the advertisement gets (statistical impact shown above). Table 17 and 18 reflect that on weekdays between 10 am to $5 \mathrm{pm}$ along with 5 to $8 \mathrm{pm}$ and 10 am to $5 \mathrm{pm}$ on weekends reflect a higher CTR

Strategy 4: The right combination of the various strategies listed can be utilized to generate a high CTR.

In order to achieve optimum results, it would be ideal to combine the research finding and employ a balanced mix of all three strategies mentioned. An example would be having an advertisement with admissions information through 
dynamic text along with a short video which is run on weekends between 10am and $5 \mathrm{pm}$. This could capture maximum users' attention and hence, impact the CTR positively.

The results found in this paper reflect and impact the strategy for digital marketing campaigns for B Schools. However, they can be generalized to some extent for other educational institutions as well, depending on the similarity of background and culture of participants. These strategies can be utilized to optimize investments made towards digital marketing and improve the Return on Investment (ROI). The format of advertisement that generate a higher CTR can be generalized for various digital marketing strategies in order to achieve the most out of the digital media landscape.

\section{Limitations and Future Direction}

While this paper encapsulates digital advertisement from a bird's eye view, it does not cover the entirety of it. Considering that the digital media experience is a holistic one, there are many additional factors that can impact the Click Through Rate (Sokolik, 2014). To delve deeper into these aspects, psychometric and experimental research can be conducted which can help to make a comprehensive framework including device and user-end principles which rule the effectiveness of the advertisement. The scope of this study is restricted to Google Adwords. As indicated in the study, the findings have examined the Indian demographics as well as Indian B Schools. The results may or may not be partially or completely applicable to the demographics of other countries, cultures and behavior. To study different audiences, a replication of the research in the specific demographic will derive the desired results. Lastly, students who wish to pursue Business and Management education have distinct behavioral and ideological characteristics which are vastly different from students pursuing other career prospects like humanities, liberal arts, life sciences, etc. The content of every advertisement studied, therefore, also stands questioned from a larger perspective as these aspirants may not necessarily be interested in pursuing the course, rather in the admissions information, faculty expertise, etc. Further research in this direction can also be conducted.

\section{Acknowledgement}

The authors of this study would like to thank National Institution Ranking Framework (NIRF) Government of India for publishing the B School Ranking which is the backbone of this research work. Selecting B Schools and analyzing the results was solely possible because of the NIRF ranking.

\section{Ethics}

This article is original and contains unpublished material. The authors confirm to have read and approved the manuscript. There are no ethical issues involved.

\section{References}

Alexa, E., Alexa, M., \& Stoica, C. (2012). The Use of Online Marketing and Social Media in Higher Education Institutions in Romania. Journal of Marketing Research and Case Studies, 1-9. https://doi.org/10.5171/2012.721221

Arora, D. N. (n.d.). ATL, BTL and TTL Marketing in Education Industry. 3.

Arora-ATL, BTL and TTL Marketing in Education Industry.pdf. (n.d.). Retrieved August 20, 2020, from http://www.ijriss.org/DigitalLibrary/Vol.2\&Issue1/13-15.pdf

Bush, V., Ferrell, O. C., \& Thomas, J. L. (1998). Marketing the Business School: An Exploratory Investigation. Journal of Marketing Education, 20(1), 16-23. https://doi.org/10.1177/027347539802000103

Chaffey, D., \& Patron, M. (2012). From web analytics to digital marketing optimization: Increasing the commercial value of digital analytics. Journal of Direct, Data and Digital Marketing Practice, 14(1), 30-45. https://doi.org/10.1057/dddmp.2012.20

Fierro, I., Arbelaez, D., \& Gavilanez, J. (2017). Digital marketing: A new tool for international education. https://doi.org/10.14482/PEGE.43.10594

Flores, L. (2013). How to Measure Digital Marketing: Metrics for Assessing Impact and Designing Success. Springer.

Geddes, B. (2014). Advanced Google AdWords. John Wiley \& Sons.

Girchenko, T., \& Ovsiannikova, Y. (2016). DIGITAL MARKETING AND ITS ROLE IN THE MODERN BUSINESS PROCESSES. European Cooperation, 11(18), 24-33. 
Haans, H., Raassens, N., \& van Hout, R. (2013). Search engine advertisements: The impact of advertising statements on click-through and conversion rates. Marketing Letters, 24(2), 151-163. https://doi.org/10.1007/s11002-013-9226-5

Harsha, P. P., \& Shah, S. J. (2011). CREATING BRAND VALUE OF HIGHER EDUCATION INSTITUTION. International Journal of Maritime Technology, 19, 152-158.

Hayes, T. J., Ruschman, D., \& Walker, M. M. (2009). Social networking as an admission tool: A case study in success. Journal of Marketing for Higher Education, 19(2), 109-124. https://doi.org/10.1080/08841240903423042

India-Internet users by age group 2020. (n.d.). Statista. Retrieved August 19, 2020, from https:/www.statista.com/statistics/751005/india-share-of-internet-users-by-age-group/

Jagadeesh, R. (2000). Assuring quality in management education: The Indian context. Quality Assurance in Education, 8(3), 110-119. https://doi.org/10.1108/09684880010372707

Jan, M. T., \& Ammari, D. (2016). Advertising online by educational institutions and students' reaction: A study of Malaysian Universities. Journal of Marketing for Higher Education, 26(2), 168-180. https://doi.org/10.1080/08841241.2016.1245232

Keelery, S. (2020, July 7). Internet usage in India-Statistics \& facts. Statista. https://www.statista.com/topics/2157/internet-usage-in-india/

Kn, M. (n.d.). Quality of Management Education in India and Reengineering Process from Traditional to Modern: An Overview. Retrieved August 17, from https://www.academia.edu/36573572/Quality_of_Management_Education_in_India_and_Reengineering_Proce ss_from_Traditional_to_Modern_An_Overview

Kumar, V., \& Raman, R. (2019). Marketing your Business School on Social Media: Analyzing Social Media Content for Adopting the Right Strategies. Journal of Computer Science, 5, 1256-1282. https://doi.org/10.3844/jcssp.2019.1256.1282

Kusumawati, A. (2019). Impact of Digital Marketing on Student Decision-Making Process of Higher Education Institution: A Case of Indonesia. Journal of E-Learning and Higher Education, 1-11. https://doi.org/10.5171/2019.267057

Martín-Santana, J. D., \& Beerli-Palacio, A. (2012). The effectiveness of web advertisement: Rectangle vs contextual banners. Online Information Review, 36(3), 420-441. https://doi.org/10.1108/14684521211241431

Mishra, C. K. (2020). Digital Marketing: Scope Opportunities and Challenges. Promotion and Marketing Communications. https://doi.org/10.5772/intechopen.92329

Nandin. (2020, July 30). New Education Policy 2020 Highlights: School and higher education to see major changes [News]. Hindustan Times. https://www.hindustantimes.com/education/new-education-policy-2020-live-updates-important-takeaways/story -yYm1QaeNyFW4uTTU3g9bJO.html

Nicholls, J., Harris, J., Morgan, E., Clarke, K., \& Sims, D. (1995). Marketing higher education: The MBA experience. International Journal of Educational Management, 9(2), 31-38. https://doi.org/10.1108/09513549510082369

Pak, B. K., Mocan, B., Yoldas, S., \& Baz, N. (2018). Development of Autonomous Intelligent System for Google Advertisement. Thirteenth International Conference on Digital Information Management (ICDIM). https://doi.org/10.1109/ICDIM.2018.8847128

Philip, J. (2008). Management education in India. Proceedings from XIII International Study \&amp; Practical Conference - Competitiveness in Information Society: BRICS-countries Experience, State University of Management, Moscow, Russia

Robinson, H., Wysocka, A., \& Hand, C. (2007). Internet advertising effectiveness. International Journal of Advertising, 26(4), 527-541. https://doi.org/10.1080/02650487.2007.11073031

Saura, J. R., Palos-Sánchez, P., \& Cerdá Suárez, L. M. (2017). Understanding the Digital Marketing Environment with KPIs and Web Analytics. Future Internet, 9(4), 76. https://doi.org/10.3390/fi9040076 
Shah, A. (2012, September). India's business schools get tough lesson in supply and demand. Reuters. Retrieved from http://in.reuters.com/article/2012/09/10/india-education-mbabusiness- schools-idINDEE88900W20120910

Shi, X. (2017). A Study on Factors Influencing the Click Through Rate, CTR, of Internet advertisement by the Charging Mode of CPM (Cost Per Mile) Based on Information Transfer. https://doi.org/10.5013/IJSSST.a.17.32.35

Sinha, D. P. (2006). Management education in India: Perspectives and challenges. Hyderabad, India: ICFAI University Press, ISBN: 9788178812793.

Smith, K. T. (2012). Longitudinal study of digital marketing strategies targeting Millennials. Journal of Consumer Marketing, 29(2), 86-92. https://doi.org/10.1108/07363761211206339

Sokolik, K., Magee, R. G., \& Ivory, J. D. (2014). Red-Hot and Ice-Cold Web Advertisement: The Influence of Web Advertisement' Warm and Cool Colors on Click-Through Rates. Journal of Interactive Advertising, 14(1), 1-7. https://doi.org/10.1080/15252019.2014.907757

Szymanski, G., \& Lininski, P. (2018). Model of the Effectiveness of Google Adwords Advertising Activities. 2018 IEEE 13th International Scientific and Technical Conference on Computer Sciences and Information Technologies (CSIT), 2, 98-101. https://doi.org/10.1109/STC-CSIT.2018.8526633

Tiago, M. T. P. M. B., \& Veríssimo, J. M. C. (2014). Digital marketing and social media: Why bother? Business Horizons, 57(6), 703-708. https://doi.org/10.1016/j.bushor.2014.07.002

Top Business Schools in 2020. (2020, February 27). Top Universities. https://www.topuniversities.com/university-rankings-articles/university-subject-rankings/top-business-schools-2 020

Turnbull, D., \& Bright, L. (2008). Advertising academia with sponsored search: An exploratory study examining the effectiveness of Google AdWords at the local and global level. Int. J. Electron. Bus. https://doi.org/10.1504/IJEB.2008.018070

University and business school ranking in India. (n.d.). Retrieved August 18, 2020, from https://eduniversal-ranking.com/business-school-university-ranking-in-india.html

University Grants commission: Genesis. (n.d.). Retrieved August 17, 2020, from https://www.ugc.ac.in/page/Genesis.aspx

Yang, X., Deng, T., Tan, W., Tao, X., Zhang, J., Qin, S., \& Ding, Z. (2019). Learning Compositional, Visual and Relational Representations for CTR Prediction in Sponsored Search. Proceedings of the 28th ACM International Conference on Information and Knowledge Management, 2851-2859. https://doi.org/10.1145/3357384.3357833

\section{Copyrights}

Copyright for this article is retained by the author(s), with first publication rights granted to the journal.

This is an open-access article distributed under the terms and conditions of the Creative Commons Attribution license (http://creativecommons.org/licenses/by/4.0/). 